IMA Journal of Numerical Analysis (2017) Page 1 of 34 doi:10.1093/imanum/drn000

\title{
Convergence analysis of high-order commutator-free quasi-Magnus exponential integrators for non-autonomous linear evolution equations of parabolic type
}

\author{
SERGio BLANES \\ Universitat Politècnica de València, Instituto de Matemática Multidisciplinar, \\ 46022 Valencia, Spain. E-mail: serblaza@imm.upv.es \\ FERNANDO CASAS \\ Universitat Jaume I, IMAC and Departament de Matemàtiques, \\ 12071 Castellón, Spain. E-mail: fernando.casas@uji.es \\ MECHTHILD ThaLHAMMER \\ Leopold-Franzens Universität Innsbruck, Institut für Mathematik, \\ Technikerstraße 13/VII, 6020 Innsbruck, Austria. \\ E-mail: mechthild.thalhammer@uibk.ac.at
}

[Received on 11 February 2017]

\begin{abstract}
The main objective of this work is to provide a stability and error analysis of high-order commutator-free quasi-Magnus (CFQM) exponential integrators. These time integration methods for non-autonomous linear evolution equations are formed by products of exponentials involving linear combinations of the defining operator evaluated at certain times. In comparison with other classes of time integration methods such as Magnus integrators, an inherent advantage of CFQM exponential integrators is that structural properties of the operator are well-preserved by the arising linear combinations. Employing the analytical framework of sectorial operators in Banach spaces, evolution equations of parabolic type and dissipative quantum systems are included in the scope of applications. In this context, however, numerical experiments show that CFQM exponential integrators of nonstiff order five or higher proposed in the literature suffer from poor stability properties. The given analysis delivers insight that CFQM exponential integrators are well-defined and stable only if the coefficients occurring in the linear combinations satisfy a positivity condition and that an alternative approach for the design of stable high-order schemes relies on the consideration of complex coefficients. Together with suitable local error expansions this implies that a high-order CFQM exponential integrator retains its nonstiff order of convergence under appropriate regularity and compatibility requirements on the exact solution. Numerical examples confirm the theoretical result and illustrate the favourable behaviour of novel schemes involving complex coefficients in stability and accuracy.
\end{abstract}

Keywords: Non-autonomous linear evolution equations, Parabolic initial-boundary value problems, Dissipative quantum systems, Exponential integrators, Magnus integrators, Commutator-free quasi-Magnus exponential integrators, Stability, Local error, Convergence

AMS: 65L05, 65M12, 65J10

(c) The author 2017. Published by Oxford University Press on behalf of the Institute of Mathematics and its Applications. All rights reserved. 


\section{Introduction}

Class of EVOlution EQUATIONS We consider evolution equations of the form

$$
\left\{\begin{array}{l}
u^{\prime}(t)=A(t) u(t), \quad t \in\left[t_{0}, T\right] \\
u\left(t_{0}\right) \text { given }
\end{array}\right.
$$

defined by a family of time-dependent linear operators $(A(t))_{t \in\left[t_{0}, T\right]}$ in a Banach space. We are primarily interested in situations where (1.1) is related to a partial differential equation of parabolic type or a dissipative quantum system, respectively. Non-autonomous linear evolution equations arise in sensitivity analysis or optimal control; further relevant applications include driven open quantum systems such as the parametrically driven dissipative Dicke model, see AlvermanN, FEHSKe, LitTlewood (2012) and references given therein.

NONLINEAR EQUATIONS AND LINEARISATIONS. As an elementary illustration, we state a onedimensional partial differential equation

$$
\partial_{t} U(x, t)=f_{2}(U(x, t)) \partial_{x x} U(x, t)+f_{1}(U(x, t)) \partial_{x} U(x, t)+f_{0}(U(x, t))+g(x, t),
$$

comprising a nonlinear diffusion term, a nonlinear advection term, a nonlinear reaction term, and an additional inhomogeneity. The time-periodic logistic reaction-diffusion equation given in PAO (2001) can be cast into the above form with

$$
f_{2}(w)=c_{3}, \quad f_{1}(w)=0, \quad f_{0}(w)=c_{0}\left(w+c_{1}\right)\left(w+c_{2}\right)
$$

and constants $c_{0}, c_{1}, c_{2}, c_{3} \in \mathbb{R}$. Associated linearised equations involve the Fréchet derivative of the second-order differential operator defining the right-hand side of the equation

$$
\begin{aligned}
F(v)= & {\left[x \mapsto f_{2}(v(x)) \partial_{x x} v(x)+f_{1}(v(x)) \partial_{x} v(x)+f_{0}(v(x))\right] } \\
F^{\prime}(v) w= & {\left[x \mapsto f_{2}^{\prime}(v(x)) \partial_{x x} v(x) w(x)+f_{2}(v(x)) \partial_{x x} w(x)\right.} \\
& \left.\quad+f_{1}^{\prime}(v(x)) \partial_{x} v(x) w(x)+f_{1}(v(x)) \partial_{x} w(x)+f_{0}^{\prime}(v(x)) w(x)\right] .
\end{aligned}
$$

For instance, the variational equation, which describes the sensitivity of the solution with respect to the reference solution, corresponds to a non-autonomous linear partial differential equation

$$
\partial_{t} u(x, t)=\alpha_{2}(x, t) \partial_{x x} u(x, t)+\alpha_{1}(x, t) \partial_{x} u(x, t)+\alpha_{0}(x, t) u(x, t)
$$

with space-time-dependent coefficient functions given by

$$
\begin{gathered}
\alpha_{2}(x, t)=f_{2}(U(x, t)), \quad \alpha_{1}(x, t)=f_{1}(U(x, t)) \\
\alpha_{0}(x, t)=f_{2}^{\prime}(U(x, t)) \partial_{x x} U(x, t)+f_{1}^{\prime}(U(x, t)) \partial_{x} U(x, t)+f_{0}^{\prime}(U(x, t)) .
\end{gathered}
$$

When rewritten as abstract differential equation, the variational equation is of the form (1.1). 
EXPONENTIAL INTEGRATORS A variety of contributions confirms that exponential time integration methods are favourable in various respects, see Alvermann, FeHSKe (2011); Alvermann, FeHSKe, LitTlewood (2012); BAder, ISERLEs, Kropielnicka, Singh (2016); Blanes, MoAN (2006); Crouch, Grossman (1993); Hochbruck, Lubich (2003); ThalHammer (2006) and references given therein. Exponential integrators for non-autonomous evolution equations commonly rely on the computation of several exponentials, which involve the values of the defining operator at certain nodes and combinations thereof. For the associated spatially semi-discretised equation, the action of the arising matrix exponentials on vectors is often advantageously realised by polynomial approximations such as Chebyshev or Krylov methods, see also HOCHBRUCK, LUBICH (1997); MOLER, VAN LOAN (2003); SIDJE (1998).

MAGNUS INTEGRATORS A well-established class of exponential time integration methods for nonautonomous linear evolution equations (1.1) is based on a formal solution representation by the Magnus expansion

$$
\begin{aligned}
u\left(t_{n+1}\right)= & \mathrm{e}^{\Omega\left(\tau_{n}, t_{n}\right)} u\left(t_{n}\right), \quad t_{0} \leqslant t_{n}<t_{n+1}=t_{n}+\tau_{n} \leqslant T \\
\Omega\left(\tau_{n}, t_{n}\right)= & \int_{t_{n}}^{t_{n}+\tau_{n}} A(\sigma) \mathrm{d} \sigma \\
& +\frac{1}{2} \int_{t_{n}}^{t_{n}+\tau_{n}} \int_{t_{n}}^{\sigma_{1}}\left[A\left(\sigma_{1}\right), A\left(\sigma_{2}\right)\right] \mathrm{d} \sigma_{2} \mathrm{~d} \sigma_{1} \\
& +\frac{1}{6} \int_{t_{n}}^{t_{n}+\tau_{n}} \int_{t_{n}}^{\sigma_{1}} \int_{t_{n}}^{\sigma_{2}}\left(\left[A\left(\sigma_{1}\right),\left[A\left(\sigma_{2}\right), A\left(\sigma_{3}\right)\right]\right]\right. \\
& \left.+\left[A\left(\sigma_{3}\right),\left[A\left(\sigma_{2}\right), A\left(\sigma_{1}\right)\right]\right]\right) \mathrm{d} \sigma_{3} \mathrm{~d} \sigma_{2} \mathrm{~d} \sigma_{1} \\
& +\cdots,
\end{aligned}
$$

see Magnus (1954); the natural approach to truncate the infinite series and to employ quadrature approximations of the arising multiple integrals leads to interpolatory Magnus integrators.

However, as has been noticed in CELLEDONI, MARTHINSEN, OWREN (2003), the use of commutators may be undesirable for solving stiff systems. In particular, in the context of partial differential equations, Magnus integrators manifest a fundamental difficulty. In consideration of the fact that the commutator

$$
\left[A\left(\sigma_{1}\right), A\left(\sigma_{2}\right)\right]=A\left(\sigma_{1}\right) A\left(\sigma_{2}\right)-A\left(\sigma_{2}\right) A\left(\sigma_{1}\right)
$$

in general does not inherit the characteristic properties of the underlying operator, one has to face the issue of well-definedness. Moreover, as relevant applications include partial differential equations in two and three space dimensions, the systems resulting from spatial semi-discretisation commonly involve large matrices; the computation of the action of discrete counterparts to iterated commutators such as

$$
\left[A\left(\sigma_{1}\right),\left[A\left(\sigma_{2}\right), A\left(\sigma_{3}\right)\right]\right]
$$

on vectors, needed for the realisation of higher-order Magnus integrators by Krylov-type methods, can be exceedingly costly due to the number of matrix-vector products required, see also BLANES, CASAS, THALHAMMER (2016) and references therein.

Nevertheless, there are situations where a special structure of the defining operator enables significant simplifications and the action of the resulting matrix-commutators on vectors can be efficiently carried out. This is the case, for instance, for the linear Schrödinger equation in the semiclassical regime with a time-dependent potential, see BADER, IsErLeS, KropielniCKA, SingH (2016). 
COMMUTATOR-FREE QUASI-MAGNUS EXPONENTIAL INTEGRATORS In the present work, we focus on a class of exponential time integration methods that circumvents the difficulties of Magnus integrators in the context of partial differential equations and thus may provide a favourable alternative, see Alvermann, Fehske (2011); Blanes, Moan (2006); Thalhammer (2006). In view of the attempt to avoid the presence of iterated commutators, we employ the notion commutator-free exponential integrator, and to distinguish them from the commutator-free exponential integrators considered in Celledoni, Marthinsen, Owren (2003); Owren (2006), we add the term quasi-Magnus.

The basic idea leading to commutator-free quasi-Magnus (CFQM) exponential integrators is to replace the single exponential (1.3) by the composition of several exponentials involving linear combinations of the values of the underlying operator at certain nodes

$$
\begin{gathered}
u_{n+1}=\mathrm{e}^{\tau_{n} B_{n J}} \ldots \mathrm{e}^{\tau_{n} B_{n 1}} \approx u\left(t_{n+1}\right)=\mathrm{e}^{\Omega\left(\tau_{n}, t_{n}\right)} u\left(t_{n}\right), \\
c_{k} \in[0,1], \quad A_{n k}=A\left(t_{n}+c_{k} \tau_{n}\right), \quad k \in\{1, \ldots, K\}, \\
B_{n j}=a_{j 1} A_{n 1}+\cdots+a_{j K} A_{n K}, \quad j \in\{1, \ldots, J\} .
\end{gathered}
$$

An inherent advantage of CFQM exponential integrators over Magnus integrators is that structural properties of the defining operator family are well-preserved by the arising linear combinations. As a consequence, well-definedness and stability of the time-discrete solution can be established for instance within the framework of sectorial operators and analytic semigroups under natural (weak) regularity requirements on the defining operator family.

EXAMPLES Henceforth, we denote by $p \in \mathbb{N}$ the nonstiff order of the method.

(i) As a first example, we mention the exponential midpoint rule of nonstiff order two

$$
p=2: \quad u_{n+1}=\mathrm{e}^{\tau_{n} A\left(t_{n}+\frac{1}{2} \tau_{n}\right)} u_{n} \approx u\left(t_{n+1}\right)=\mathrm{e}^{\Omega\left(\tau_{n}, t_{n}\right)} u\left(t_{n}\right),
$$

which is an instance of a Magnus integrator and likewise fits into the class of CFQM exponential integrators.

(ii) A Magnus integrator of nonstiff order four is based on two Gaussian nodes

$$
\begin{gathered}
p=4: \quad u_{n+1}=\mathrm{e}^{\tau_{n} a_{1}\left(A_{n 1}+A_{n 2}\right)+\tau_{n}^{2} a_{2}\left[A_{n 2}, A_{n 1}\right]} u_{n} \approx u\left(t_{n+1}\right)=\mathrm{e}^{\Omega\left(\tau_{n}, t_{n}\right)} u\left(t_{n}\right), \\
c_{1}=\frac{1}{2}-\frac{\sqrt{3}}{6}, \quad c_{2}=\frac{1}{2}+\frac{\sqrt{3}}{6}, \quad a_{1}=\frac{1}{2}, \quad a_{2}=\frac{\sqrt{3}}{12} .
\end{gathered}
$$

A related CFQM exponential integrator of nonstiff order four is given by

$$
\begin{aligned}
& p=4: \quad u_{n+1}=\mathrm{e}^{\tau_{n}\left(a_{21} A_{n 1}+a_{22} A_{n 2}\right)} \mathrm{e}^{\tau_{n}\left(a_{11} A_{n 1}+a_{12} A_{n 2}\right)} u_{n} \approx u\left(t_{n+1}\right)=\mathrm{e}^{\Omega\left(\tau_{n}, t_{n}\right)} u\left(t_{n}\right), \\
& c_{1}=\frac{1}{2}-\frac{\sqrt{3}}{6}, \quad c_{2}=\frac{1}{2}+\frac{\sqrt{3}}{6}, \quad a_{11}=a_{22}=\frac{1}{4}+\frac{\sqrt{3}}{6}, \quad a_{12}=a_{21}=\frac{1}{4}-\frac{\sqrt{3}}{6} .
\end{aligned}
$$

RELATED CLASSES OF COMMUTATOR-FREE EXPONENTIAL INTEGRATORS It is worth mentioning the connection of CFQM exponential integrators to other classes of commutator-free exponential integrators.

In Celledoni, MARThinsen, Owren (2003), autonomous nonlinear (ordinary) differential equations of the form

$$
u^{\prime}(t)=F(u(t))=\sum_{\ell=1}^{L} f_{\ell}(u(t)) E_{\ell}(u(t))
$$


are studied; the family of vector fields $\left(E_{\ell}\right)_{\ell=1}^{L}$ is assumed to span, at every point, the tangent space, and $\left(f_{\ell}\right)_{\ell=1}^{L}$ denotes a family of real or complex-valued functions defined on the underlying manifold. Under the assumption that for a fixed value $y$ the differential equation

$$
w^{\prime}(t)=\sum_{\ell=1}^{L} f_{\ell}(y) E_{\ell}(w(t))
$$

is more easily solvable than the original one, explicit commutator-free exponential integrators of orders three and four involving three and five exponentials, respectively, are proposed.

A non-autonomous linear equation of the form (1.1) can be recast in this way by adding the time as a coordinate, i.e., by considering the system

$$
\left\{\begin{array}{l}
u^{\prime}(t)=A(\zeta(t)) u(t) \\
\zeta^{\prime}(t)=1
\end{array}\right.
$$

However, compared to CFQM exponential integrators, this approach leads to more costly schemes, requiring for instance five exponentials for order four; besides, we are not aware of schemes of order five or higher that have been proposed in the literature.

A simpler alternative consists in considering a $p$ th-order splitting method that is defined by real or complex coefficients $\left(a_{\ell}, b_{\ell}\right)_{\ell=1}^{L}$; for non-commutative matrices $X, Y$, this in particular implies

$$
\prod_{\ell=1}^{L}\left(\mathrm{e}^{\tau a_{\ell} X} \mathrm{e}^{\tau b_{\ell} Y}\right)=\mathrm{e}^{\tau(X+Y)}+\mathscr{O}\left(\tau^{p+1}\right)
$$

The application to the autonomous system (1.6), more precisely, to the corresponding subproblems

$$
\left\{\begin{array} { l } 
{ u ^ { \prime } ( t ) = A ( \zeta ( t ) ) u ( t ) , } \\
{ \zeta ^ { \prime } ( t ) = 0 , }
\end{array} \quad \left\{\begin{array}{l}
u^{\prime}(t)=0, \\
\zeta^{\prime}(t)=1,
\end{array}\right.\right.
$$

leads to the commutator-free exponential integrator

$$
u_{n+1}=\prod_{\ell=1}^{L} \mathrm{e}^{\tau_{n} a_{\ell} A\left(t_{n}+c_{\ell} \tau_{n}\right)} u_{n}, \quad c_{\ell}=\sum_{j=1}^{\ell} b_{j}, \quad \ell \in\{1, \ldots, L\} .
$$

Again, compared to CFQM exponential integrators, the number of exponentials grows considerably with the order; moreover, for order greater than two, schemes with real coefficients necessarily involve negative coefficients and thus have poor stability properties for evolution equations of parabolic type.

OBJECTIVE AND APPROACH In this work, our main concern is to provide a stability and error analysis of high-order CFQM exponential integrators for the time integration of non-autonomous linear evolution equations of parabolic type. For this purpose, we employ the analytical framework of sectorial operators in Banach spaces.

Preliminary numerical tests for an elementary parabolic equation showed that CFQM exponential integrators of nonstiff orders six proposed in the literature suffer from poor stability properties, and a first theoretical analysis delivered insight that the structural quality of CFQM exponential integrators is only preserved under a positivity condition on certain combinations of the coefficients. Additional numerical 
tests confirmed a conjectured order barrier at order five for schemes involving real coefficients, and the connection to operator splitting method suggested to circumvent this order barrier by the consideration of complex coefficients.

On the basis of these findings, we deduce stability and local error estimates with respect to the norm of the underlying Banach space, which imply that a CFQM exponential integrator retains its nonstiff order of convergence, provided that the exact solution to the considered evolution equation satisfies suitable regularity and compatibility requirements. Numerical results obtained for a variational equation associated with a test equation of the form (1.2) confirm the theoretical convergence estimate and illustrate the favourable behaviour of novel schemes involving complex coefficients in stability and accuracy. The class of methods considered in this paper thus has good potential for its use in the time integration of non-autonomous linear evolution equations.

RELATED WORK Our analysis of CFQM exponential integrators extends former work on a fourthorder scheme within this class involving two exponentials; however, in THALHAMmER (2006), the main objective was to explain order reductions encountered for parabolic equations under homogeneous Dirichlet boundary conditions, and thus only few expansion steps of the local error were needed. The main original contribution of the present work is the accomplishment of a suitable local error expansion, applicable to the whole class of CFQM exponential integrators. The main novel aspect of the stability analysis is to include complex coefficients and to identify the positivity condition on certain linear combinations of coefficients.

The observation that high-order CFQM exponential integrators given in the literature involve negative coefficients and thus suffer from poor stability properties when applied to dissipative equations of parabolic type motivates the design of novel optimised schemes with complex coefficients. Our related work Blanes, CASAS, ThalHAMmer (2016) is concerned with this question; in particular, a set of independent conditions for time-symmetric methods of order six is given there. In addition, the efficiency of the novel schemes is compared to other time integration methods such as Magnus integrators for a dissipative problem in quantum mechanics.

OUTLINE The present manuscript is organised as follows. The class of CFQM exponential integrators is introduced in Section 2. A rigorous stability and error analysis of high-order CFQM exponential integrators is provided in Section 3. For better readability, the details of the somewhat long-winded local error expansion are included in the appendix; as an illustration of the general case, the expansion obtained for a fourth-order CFQM exponential integrator involving two nodes and two exponentials per time step and a MAPLE implementation of the resulting nonstiff order conditions are also included there. Numerical examples that confirm and complement the theoretical considerations are given in Section 4.

Notation Let $\mathbb{N}=\{n \in \mathbb{Z}: n \geqslant 0\}$ denote the set of non-negative integer numbers. For a composition of non-commutative operators, we employ the convenient short notation

$$
\prod_{i=\ell}^{m} E_{i}=\left\{\begin{array}{ll}
E_{m} \cdots E_{\ell}, & \ell \leqslant m, \\
I, & m<\ell,
\end{array} \quad \ell, m \in \mathbb{N} ;\right.
$$

here, by definition, the empty product is equal to the identity operator. Thoughout, $C>0$ denotes a generic constant. For simplicity, we do not distinguish the solutions to a partial differential equation and to the associated abstract differential equation in notation, and likewise for the defining operators. 


\section{Commutator-free quasi-Magnus exponential integrators}

In this section, we introduce the general format of CFQM exponential integrators for the nonautonomous linear evolution equation (1.1) and specify higher-order schemes.

GENERAL FORMAT As usual in a time-stepping approach, we consider suitably chosen time grid points $t_{0}<t_{1}<\cdots<t_{N}=T$ and denote by

$$
\tau_{n}=t_{n+1}-t_{n}, \quad n \in\{0,1, \ldots, N-1\},
$$

the associated time increments; throughout, we employ the standard assumption that the ratios of subsequent time stepsizes are bounded from below and above

$$
\rho_{\min } \leqslant \frac{\tau_{n+1}}{\tau_{n}} \leqslant \rho_{\max }, \quad n \in\{0,1, \ldots, N-2\} .
$$

For a given initial approximation, the time-discrete solution values are determined by recurrence

$$
u_{0} \approx u\left(t_{0}\right), \quad u_{n+1}=S_{n}\left(\tau_{n}\right) u_{n} \approx u\left(t_{n+1}\right), \quad n \in\{0,1, \ldots, N-1\} ;
$$

a high-order CFQM exponential integrator can be cast into the format

$$
\begin{gathered}
S_{n}\left(\tau_{n}\right)=\prod_{j=1}^{J} \mathrm{e}^{\tau_{n} B_{n j}}, \\
\mathscr{J}=\{1, \ldots, J\}, \quad \mathscr{K}=\{1, \ldots, K\}, \\
A_{n k}=A\left(t_{n}+c_{k} \tau_{n}\right), \quad B_{n j}=\sum_{k=1}^{K} a_{j k} A_{n k}, \quad(j, k) \in \mathscr{J} \times \mathscr{K} .
\end{gathered}
$$

As common, we relate the nodes to quadrature nodes which we assume to be contained in the unit interval and monotonically increasing

$$
0 \leqslant c_{1}<\cdots<c_{K} \leqslant 1
$$

for evolution equations of parabolic type, it is beneficial to permit complex coefficients in the linear combinations. Henceforth, we use the abbreviations

$$
b_{j}=\sum_{k=1}^{K} a_{j k}, \quad d_{j}=\sum_{\ell=1}^{j} b_{\ell}, \quad \gamma_{j \ell}=\sum_{k=1}^{K} a_{j k} c_{k}^{\ell}, \quad j \in \mathscr{J}, \quad \ell \in \mathbb{N},
$$

and set $d_{0}=0$ as well as $d_{0}^{0}=1$. For a time-independent operator $A$, we have

$$
S_{n}\left(\tau_{n}\right)=\prod_{j=1}^{J} \mathrm{e}^{\tau_{n} b_{j} A},
$$

which explains the basic necessity of the positivity condition

$$
\Re b_{j}>0, \quad j \in \mathscr{J}
$$


indeed, this elemental requirement ensures that a CFQM exponential integrator remains well-defined within the analytical framework of sectorial operators and analytic semigroups, see also Section 3. Moreover, we tacitly assume that the consistency condition

$$
d_{J}=\sum_{j=1}^{J} b_{j}=\sum_{j=1}^{J} \sum_{k=1}^{K} a_{j k}=1
$$

is satisfied; this relation is a direct consequence of the basic requirement $S_{n}\left(\tau_{n}\right)=\mathrm{e}^{\tau_{n} A}$ for a timeindependent operator $A$. We recall that $p \in \mathbb{N}$ denotes the nonstiff order of the method.

EXAMPLES As illustration and in view of numerical comparisons described in Section 4, we recall the CFQM exponential integrators introduced before and specify the coefficients of fifth- and sixth-order schemes. For further examples, among them optimised schemes with small effective error constants, we refer to Alvermann, Fehske (2011); Alvermann, Fehske, Littlewood (2012); Blanes, CASAS, OTEO, Ros (2009); Blanes, MOAN (2006).

Often, the coefficient matrix $a \in \mathbb{C}^{J \times K}$ is defined by the Gaussian nodes and weights $\left(c_{i}, w_{i}\right)_{i=1}^{K}$, which correspond to a quadrature approximation of maximum order $2 K$. In some situations, however, the use of a different quadrature formula of the same order or higher may be convenient or favourable, providing more accurate approximations without considerably increasing the computational cost. The new coefficient matrix related to quadrature nodes and weights $\left(\widehat{c}_{i}, \widehat{w}_{i}\right)_{i=1}^{\widehat{K}}$ is given by

$$
\begin{aligned}
& \widehat{a}=a\left(Q^{[K, K]}\right)^{-1} \widehat{Q}^{[K, \widehat{K}]}, \\
& Q^{[K, K]} \in \mathbb{R}^{K \times K}, \quad Q_{i j}^{[K, K]}=w_{j}\left(c_{j}-\frac{1}{2}\right)^{i-1}, \quad i, j \in\{1, \ldots, K\}, \\
& \widehat{Q}^{[K, \widehat{K}]} \in \mathbb{R}^{K \times \widehat{K}}, \quad \widehat{Q}_{i j}^{[K, \widehat{K}]}=\widehat{w}_{j}\left(\widehat{c}_{j}-\frac{1}{2}\right)^{i-1}, \quad i \in\{1, \ldots, K\}, \quad j \in\{1, \ldots, \widehat{K}\} .
\end{aligned}
$$

We note that the row sums of $a$ are equal to the row sums of $\widehat{a}$, that is, the validity of condition (2.3c) is independent of the underlying quadrature formula.

(i) Order 2. The exponential midpoint rule (1.4) is based on a single Gaussian node and involves a single exponential at each time step

$$
p=2, \quad J=K=1, \quad c_{1}=\frac{1}{2}, \quad w_{1}=1, \quad a_{11}=1 ;
$$

evidently, condition (2.3c) is satisfied. Considering instead the trapezoidal rule, we obtain

$$
\widehat{c}_{1}=0, \quad \widehat{c}_{2}=1, \quad \widehat{w}_{1}=\widehat{w}_{2}=\frac{1}{2}, \quad \widehat{a}_{11}=\widehat{a}_{12}=\frac{1}{2} .
$$

(ii) Order 4. The fourth-order CFQM exponential integrator (1.5) is based on the Gaussian quadrature formula and requires the evaluation of two exponentials at each time step

$$
\begin{aligned}
p=4, \quad J=K=2, \quad & c_{1}=\frac{1}{2}-\frac{\sqrt{3}}{6}, \quad c_{2}=\frac{1}{2}+\frac{\sqrt{3}}{6}, \quad w_{1}=w_{2}=\frac{1}{2}, \\
a & =\left(\begin{array}{ll}
\frac{1}{4}+\frac{\sqrt{3}}{6} & \frac{1}{4}-\frac{\sqrt{3}}{6} \\
\frac{1}{4}-\frac{\sqrt{3}}{6} & \frac{1}{4}+\frac{\sqrt{3}}{6}
\end{array}\right) ;
\end{aligned}
$$

in particular, condition (2.3c) is satisfied, since

$$
b_{1}=a_{11}+a_{12}=\frac{1}{2}, \quad b_{2}=a_{21}+a_{22}=\frac{1}{2} .
$$


For instance, for the Simpson rule, we obtain

$$
\begin{gathered}
\widehat{c}_{1}=0, \quad \widehat{c}_{2}=\frac{1}{2}, \quad \widehat{c}_{3}=1, \quad \widehat{w}_{1}=\widehat{w}_{3}=\frac{1}{6}, \quad \widehat{w}_{2}=\frac{2}{3}, \\
\widehat{a}=\frac{1}{12}\left(\begin{array}{ccc}
3 & 4 & -1 \\
-1 & 4 & 3
\end{array}\right) ;
\end{gathered}
$$

for the sixth-order Gaussian quadrature formula, we have

$$
\begin{gathered}
\widehat{c}_{1}=\frac{1}{2}-\sqrt{\frac{3}{20}}, \quad \widehat{c}_{2}=\frac{1}{2}, \quad \widehat{c}_{3}=\frac{1}{2}+\sqrt{\frac{3}{20}}, \quad \widehat{w}_{1}=\widehat{w}_{3}=\frac{5}{18}, \quad \widehat{w}_{2}=\frac{4}{9}, \\
\widehat{a}=\left(\begin{array}{ccc}
\frac{5+2 \sqrt{15}}{36} & \frac{2}{9} & \frac{5-2 \sqrt{15}}{36} \\
\frac{5-2 \sqrt{15}}{36} & \frac{2}{9} & \frac{5+2 \sqrt{15}}{36}
\end{array}\right) .
\end{gathered}
$$

(iii) Order 5. A fifth-order CFQM exponential integrator with complex coefficients that satisfies condition (2.3c) is designed in Blanes, CASAS, ThalHammer (2016). Employing a quadrature approximation based on the sixth-order Gaussian quadrature rule, see (2.5), the coefficient matrix reads

$$
\begin{gathered}
p=5, \quad J=K=3, \\
a=\left(\begin{array}{ccc}
\frac{145+37 \sqrt{15}}{900}+\frac{5+3 \sqrt{15}}{300} \mathrm{i} & -\frac{1}{45}+\frac{1}{15} \mathrm{i} & \frac{145-37 \sqrt{15}}{900}+\frac{5-3 \sqrt{15}}{300} \mathrm{i} \\
-\frac{2}{45}-\frac{\sqrt{15}}{50} \mathrm{i} & \frac{22}{45} & -\frac{2}{45}+\frac{\sqrt{15}}{50} \mathrm{i} \\
\frac{145-37 \sqrt{15}}{900}-\frac{5-3 \sqrt{15}}{300} \mathrm{i} & -\frac{1}{45}-\frac{1}{15} \mathrm{i} & \frac{145+37 \sqrt{15}}{900}-\frac{5+3 \sqrt{15}}{300} \mathrm{i}
\end{array}\right) ;
\end{gathered}
$$

in particular, this implies

$$
\Re b_{1}=\mathfrak{R} b_{3}=\frac{3}{10}, \quad b_{2}=\frac{2}{5} .
$$

(iv) Order 6. A non-optimised sixth-order CFQM exponential integrator results from the coefficients $\left(f_{j k}\right)_{j, k=1}^{3}$ given in (Alvermann, FEHSKe, 2011, Table 3, CF6:6); using again the sixth-order Gaussian quadrature rule yields

$$
\begin{aligned}
& p=6, \quad J=6, \quad K=3, \\
& f=\left(\begin{array}{rrr}
0.160000000000000 & -0.151015389377465 & 0.133046168132396 \\
-0.227381647426963 & 0.087654259755115 & 0.069919836812657 \\
0.567381647426963 & -0.210351545122098 & -0.202966004945053
\end{array}\right) \text {, } \\
& F=\left(\begin{array}{rrr}
f_{11} & f_{12} & f_{13} \\
f_{21} & f_{22} & f_{23} \\
f_{31} & f_{32} & f_{33} \\
f_{31} & -f_{32} & f_{33} \\
f_{21} & -f_{22} & f_{23} \\
f_{11} & -f_{12} & f_{13}
\end{array}\right), \quad G=\left(\begin{array}{ccc}
1 & 0 & 0 \\
0 & 6 & 0 \\
-\frac{5}{2} & 0 & 30
\end{array}\right), \quad a=F G Q^{[3,3]}, \\
& a=\left(\begin{array}{rrr}
0.215838996975768 & -0.076717964591551 & 0.020878967615784 \\
-0.080897796320853 & -0.178747217537158 & 0.032263366431047 \\
0.180628460055830 & 0.477687404350931 & -0.090934216979798 \\
-0.090934216979798 & 0.477687404350931 & 0.180628460055830 \\
0.032263366431047 & -0.178747217537158 & -0.080897796320853 \\
0.020878967615784 & -0.076717964591551 & 0.215838996975768
\end{array}\right) \text {. }
\end{aligned}
$$


We point out that condition (2.3c) does not hold, since

$$
b_{1}=b_{6}=0.16, \quad b_{2}=b_{5}=-0.227381647426963, \quad b_{3}=b_{4}=0.567381647426963 ;
$$

thus, a poor stability behaviour is observed for evolution equations of parabolic type. As alternative, we introduce a sixth-order CFQM exponential integrator with complex coefficients, see Blanes, Casas, Thalhammer (2016); when based on the Gaussian nodes (2.5), the scheme is given by

$$
\begin{gathered}
p=6, \quad J=5, \quad K=3, \quad a=\Re a+\Im a, \\
\Re a=\left(\begin{array}{rrr}
0.194217945883438 & -0.056316450736459 & 0.014749454957822 \\
0.103849953683652 & 0.155323390036559 & -0.032809068534171 \\
-0.002230508212962 & 0.246430565844245 & -0.002230508212962 \\
-0.032809068534171 & 0.155323390036559 & 0.103849953683652 \\
0.014749454957822 & -0.056316450736459 & 0.194217945883438
\end{array}\right), \\
\Im a=\left(\begin{array}{rrr}
0.032784503082251 & -0.002894852021076 & 0.000390316102524 \\
-0.032105649424546 & 0.056238557581740 & -0.007595658537257 \\
0.006526488777028 & -0.106687411121327 & 0.006526488777028 \\
-0.007595658537257 & 0.056238557581740 & -0.032105649424546 \\
0.000390316102524 & -0.002894852021076 & 0.032784503082251
\end{array}\right),
\end{gathered}
$$

which in particular implies

$$
\begin{gathered}
\Re b_{1}=\Re b_{5}=0.1526509501048, \quad \Re b_{2}=\Re b_{4}=0.22636427518604, \\
\Re b_{3}=0.241969549418321 .
\end{gathered}
$$

\section{Convergence analysis}

In this section, we establish a convergence result for high-order CFQM exponential integrators applied to non-autonomous linear evolution equations of parabolic type. In Section 3.1, we specify our hypotheses on the defining operator and recapitulate basic results related to sectorial operators in Banach spaces; for details on the employed analytical framework, we refer to the standard works ENGEL, NAGEL (2000); HENRY (1981); PAZY (1983); TANABE (1979) and in particular to the monograph LunARDI (1995). An elementary initial-boundary-value problem introduced in Section 3.2 serves as illustration and test problem for numerical comparisons. A fundamental stability estimate and a suitable local error expansion are stated in Sections 3.3 and 3.4. Our main result is finally given in Section 3.5.

\subsection{Analytical framework}

As before, we let $\left(X,\|\cdot\|_{X}\right)$ denote the underlying Banach space and assume that the Banach space $\left(D,\|\cdot\|_{D}\right)$ is dense and continuously embedded in $X$. For some exponent $\mu \in(0,1)$, the associated intermediate space $D \subset X_{\mu} \subset X$ satisfies a bound of the form

$$
\|x\|_{X_{\mu}} \leqslant C\|x\|_{D}^{\mu}\|x\|_{X}^{1-\mu}, \quad x \in D
$$

as common, we set $X_{0}=X$ and $X_{1}=D$. Examples for intermediate spaces are real interpolation spaces or fractional power spaces, see HENRY (1981); LUNARDI (1995); PAZY (1983). We employ the following hypotheses on the family $(A(t))_{t \in\left[t_{0}, T\right]}$ defining the right-hand side of (1.1), see (LUNARDI, 1995, Ch. 6.1). 
Hypothesis 1 (i) The linear operator $A(t): D \rightarrow X$ is sectorial, uniformly in $t \in\left[t_{0}, T\right]$; that is, there exist constants $a \in \mathbb{R}, \phi \in\left(0, \frac{\pi}{2}\right)$, and $C_{1}>0$ such that the resolvent estimate

$$
\left\|(\lambda I-A(t))^{-1}\right\|_{X \leftarrow X} \leqslant \frac{C_{1}}{|\lambda-a|}
$$

holds for all $t \in\left[t_{0}, T\right]$ and complex numbers $\lambda \in \mathbb{C} \backslash S_{\phi}(a)$ in the complement of the sector

$$
S_{\phi}(a)=\{a\} \cup\{\lambda \in \mathbb{C}:|\arg (a-\lambda)| \leqslant \phi\} .
$$

(ii) The graph norm of $A(t)$ and the norm in $D$ are equivalent for any $t \in\left[t_{0}, T\right]$; that is, there exists a constant $C_{2}>0$ such that the relation

$$
C_{2}^{-1}\|x\|_{D} \leqslant\|x\|_{X}+\|A(t) x\|_{X} \leqslant C_{2}\|x\|_{D}, \quad x \in D
$$

is valid for all $t \in\left[t_{0}, T\right]$.

(iii) The regularity requirement $A \in \mathscr{C}^{\vartheta}\left(\left[t_{0}, T\right], L(D, X)\right)$ holds for some $\vartheta \in(0,1]$; that is, there exists a constant $C_{3}>0$ such that the bound

$$
\|A(t)-A(s)\|_{X \leftarrow D} \leqslant C_{3}(t-s)^{\vartheta}
$$

is valid for all $s, t \in\left[t_{0}, T\right]$.

Let $t \in\left[t_{0}, T\right]$. Under the above assumptions, the sectorial operator $A(t): D \rightarrow X$ generates an analytic semigroup $\left(\mathrm{e}^{\sigma A(t)}\right)_{\sigma \geqslant 0}$, given by the integral formula of Cauchy

$$
\mathrm{e}^{\sigma A(t)}= \begin{cases}\frac{1}{2 \pi \mathrm{i}} \int_{\Gamma} \mathrm{e}^{\lambda}(\lambda I-\sigma A(t))^{-1} \mathrm{~d} \lambda, & \sigma>0, \\ I, & \sigma=0,\end{cases}
$$

for some path $\Gamma$ surrounding the spectrum of $A(t)$; the resolvent estimate (3.2a) ensures that the operator $\mathrm{e}^{\sigma A(t)}: X \rightarrow X$ remains bounded, see also relation (3.3) below.

In order to show well-definedness and stability of high-order CFQM exponential integrators, we make use of the following auxiliary considerations, see also HENRY (1981); LUNARDI (1995); PAZY (1983).

Remark 1 Let $t \in\left[t_{0}, T\right]$.

(i) For any $\tau>0$ and $\lambda \in \mathbb{C} \backslash S_{\phi}(a)$, the resolvent bound implies

$$
\left\|(\lambda I-\tau A(t))^{-1}\right\|_{X \leftarrow X}=\frac{1}{\tau}\left\|\left(\frac{1}{\tau} \lambda I-A(t)\right)^{-1}\right\|_{X \leftarrow X} \leqslant \frac{C_{1}}{|\lambda-\tau a|} .
$$


By the required equivalence of the graph norm and the norm in $D$, this yields the estimate

$$
\begin{aligned}
\left\|(\lambda I-\tau A(t))^{-1}\right\|_{D \leftarrow D} & =\sup _{\|x\|_{D}=1}\left\|(\lambda I-\tau A(t))^{-1} x\right\|_{D} \\
& \leqslant C_{2} \sup _{\|x\|_{D}=1}\left(\left\|(\lambda I-\tau A(t))^{-1} x\right\|_{X}+\left\|A(t)(\lambda I-\tau A(t))^{-1} x\right\|_{X}\right) \\
& \leqslant C_{2}\left\|(\lambda I-\tau A(t))^{-1}\right\|_{X \leftarrow X} \sup _{\|x\|_{D}=1}\left(\|x\|_{X}+\|A(t) x\|_{X}\right) \\
& \leqslant C_{2}^{2}\left\|(\lambda I-\tau A(t))^{-1}\right\|_{X \leftarrow X} \\
& \leqslant \frac{C_{1} C_{2}^{2}}{|\lambda-\tau a|} .
\end{aligned}
$$

Moreover, together with the identity $\tau A(t)(\lambda I-\tau A(t))^{-1}=\lambda(\lambda I-\tau A(t))^{-1}-I$, the bound

$$
\begin{aligned}
\tau\left\|(\lambda I-\tau A(t))^{-1}\right\|_{D \leftarrow X} & =\sup _{\|x\|_{X}=1} \tau\left\|(\lambda I-\tau A(t))^{-1} x\right\|_{D} \\
& \leqslant C_{2} \sup _{\|x\|_{X}=1}\left(\tau\left\|(\lambda I-\tau A(t))^{-1} x\right\|_{X}+\tau\left\|A(t)(\lambda I-\tau A(t))^{-1} x\right\|_{X}\right) \\
& \leqslant C_{2}\left(\tau\left\|(\lambda I-\tau A(t))^{-1}\right\|_{X \leftarrow X}+\left\|\lambda(\lambda I-\tau A(t))^{-1}-I\right\|_{X \leftarrow X}\right) \\
& \leqslant C_{2}\left(1+\frac{C_{1}(\tau+|\lambda|)}{|\lambda-\tau a|}\right)
\end{aligned}
$$

follows.

(ii) Let $T_{\sigma}>0$. The above considerations lead to a basic estimate for the analytic semigroup

$$
\left\|\mathrm{e}^{\sigma A(t)}\right\|_{X \leftarrow X}+\left\|\mathrm{e}^{\sigma A(t)}\right\|_{D \leftarrow D}+\sigma\left\|\mathrm{e}^{\sigma A(t)}\right\|_{D \leftarrow X} \leqslant C, \quad \sigma \in\left[0, T_{\sigma}\right] ;
$$

more generally, for exponents $0 \leqslant \mu \leqslant v \leqslant 1$ and $k \in \mathbb{N}$, we obtain

$$
\sigma^{k+v-\mu}\left\|(A(t))^{k} \mathrm{e}^{\sigma A(t)}\right\|_{X_{v} \leftarrow X_{\mu}} \leqslant C, \quad \sigma \in\left[0, T_{\sigma}\right]
$$

see also (3.1).

\subsection{Illustration}

Let $\Omega \subset \mathbb{R}$ denote a bounded closed interval. In regard to (1.2), we consider the initial value problem for a non-autonomous linear partial differential equation

$$
\left\{\begin{array}{l}
\partial_{t} u(x, t)=\alpha_{2}(x, t) \partial_{x x} u(x, t)+\alpha_{1}(x, t) \partial_{x} u(x, t)+\alpha_{0}(x, t) u(x, t), \quad(x, t) \in \Omega \times\left[t_{0}, T\right], \\
u(x, 0)=u_{0}(x), \quad x \in \Omega
\end{array}\right.
$$

Under the basic assumption that all values of the leading space-time-dependent coefficient function are positive, the equation is of parabolic type. In order to rewrite (3.4) as an abstract Cauchy problem of the form (1.1), we choose the space of continuous functions as underlying Banach space

$$
\begin{gathered}
X=\mathscr{C}(\Omega, \mathbb{R}), \quad D=\mathscr{C}^{2}(\Omega, \mathbb{R}), \\
A(t): D \longrightarrow X: w \longmapsto\left[x \mapsto \alpha_{2}(x, t) \partial_{x x} w(x)+\alpha_{1}(x, t) \partial_{x} w(x)+\alpha_{0}(x, t) w(x)\right], \quad t \in\left[t_{0}, T\right] .
\end{gathered}
$$


Further restrictions apply when additional boundary conditions are imposed; for instance, in the case of homogeneous Dirichlet boundary conditions, the domain of the defining operator is given by

$$
D=\left\{w \in \mathscr{C}^{2}(\Omega, \mathbb{R}):\left.w(x)\right|_{x \in \partial \Omega}=0\right\} .
$$

Analogous considerations are valid for alternative choices such as $X=L^{2}(\Omega, \mathbb{R})$.

For a detailed exposition and the natural extension to several space dimensions, we refer to (LunARDI, 1995, Ch. 3). Provided that the boundary of the spatial domain $\Omega \subset \mathbb{R}^{d}$ is sufficiently regular and that the (complex-valued) space-time-dependent coefficient functions are sufficiently smooth with respect to the spatial variables, it is for instance shown that a (strongly) elliptic second-order differential operator under homogeneous boundary conditions generates a sectorial operator in the space of continuous functions, see (LUNARDI, 1995, Corollary 3.1.21). Furthermore, assuming the coefficient functions to be Hölder-continuous with respect to time permits to extend fundamental results for the time-independent case to the time-dependent case, see (LuNARDI, 1995, Ch. 6). We point out that the construction of the evolution operator associated with a non-autonomous linear equation and in particular the proof of its well-definedness as operator from $X$ to $X$ is a non-trivial task; the stability bound given in Section 3.3 conforms to the first statement in (LuNARDI, 1995, Corollary 6.1.8).

\subsection{Well-definedness and stability}

Employing the analytical framework introduced in Section 3.1, it is ensured that high-order CFQM exponential integrators remain well-defined on the underlying Banach space.

Remark 2 Let $n \in\{0,1, \ldots, N-1\}$ and $j \in \mathscr{J}$. Under Hypothesis 1 on the linear operator family and assumption (2.3) on the method coefficients, the operator $B_{n j}: D \rightarrow X$ defining the CFQM exponential integrator (2.2) is sectorial; indeed, employing the reformulation

$$
B_{n j}=\sum_{k=1}^{K} a_{j k} A\left(t_{n}+c_{k} \tau\right)=b_{j} A\left(t_{n}\right)+\sum_{k=1}^{K} a_{j k}\left(A\left(t_{n}+c_{k} \tau\right)-A\left(t_{n}\right)\right)
$$

noting that the operator $b_{j} A\left(t_{n}\right)$ is sectorial if $\Re b_{j}>0$ and that the remainder is bounded by the Hölder continuity assumption (3.2c), the statement follows from a perturbation result for sectorial operators (PAZY, 1983, Sec. 3.2).

A stability bound for high-order CFQM exponential integrators is provided by the following result.

Theorem 1 (Stability) Let $n_{0}, n \in\{0,1, \ldots, N-1\}$ be such that $n_{0} \leqslant n$. Under Hypothesis 1 , assumption (2.1) on the sequence of time stepsizes, and condition (2.3c) on the method coefficients, the time-discrete evolution operator associated with a high-order CFQM exponential integrator (2.2) satisfies the following bound with a constant $C>0$ that depends on $t_{N}=T$, in general, but is independent of $N$ and the stepsize sequence

$$
\left\|\prod_{i=n_{0}}^{n} S_{i}\left(\tau_{i}\right)\right\|_{X \leftarrow X} \leqslant C .
$$

Proof. We recall that $C>0$ denotes a generic constant which possibly has different values at different occurrences. Without loss of generality, we henceforth assume $n \gg n_{0}$; otherwise, the boundedness of compositions of the time-discrete evolution operator follows at once from a repeated application of (3.3). Our proof of the above stability bound is in the lines of the preceding work THALHAMMER 
(2006), see also references given therein. The basic idea is to use the relations

$$
\begin{gathered}
\prod_{i=n_{0}}^{n} \prod_{j=1}^{J} \mathrm{e}^{b_{j} \tau_{i} A(t)}=\mathrm{e}^{\left(t_{n+1}-t_{n_{0}}\right) A(t)}, \\
\left\|\mathrm{e}^{\left(t_{n+1}-t_{n_{0}}\right) A(t)}\right\|_{X \leftarrow X}+\left(t_{n+1}-t_{n_{0}}\right)\left\|\mathrm{e}^{\left(t_{n+1}-t_{n_{0}}\right) A(t)}\right\|_{D \leftarrow X} \leqslant C,
\end{gathered}
$$

that are valid for the analytic semigroup generated by the sectorial operator $A(t)$, due to the consistency condition (2.3) and the bounds in (3.3); for reason which will become clear subsequently, we choose $t=t_{n_{0}}+c_{K} \tau_{n_{0}}$. Thus, it suffices to estimate the difference

$$
\Delta_{n_{0}}^{n}=\prod_{i=n_{0}}^{n} S_{i}\left(\tau_{i}\right)-\mathrm{e}^{\left(t_{n+1}-t_{n_{0}}\right) A(t)} .
$$

We employ the telescopic identity

$$
\begin{aligned}
\Delta_{n_{0}}^{n} & =\sum_{i=n_{0}}^{n}\left(\prod_{\ell=i+1}^{n} S_{\ell}\left(\tau_{\ell}\right)\right)\left(S_{i}\left(\tau_{i}\right)-\mathrm{e}^{\tau_{i} A(t)}\right) \mathrm{e}^{\left(t_{i}-t_{n_{0}}\right) A(t)} \\
& =\sum_{i=n_{0}}^{n-1} \Delta_{i+1}^{n}\left(S_{i}\left(\tau_{i}\right)-\mathrm{e}^{\tau_{i} A(t)}\right) \mathrm{e}^{\left(t_{i}-t_{n_{0}}\right) A(t)}+\sum_{i=n_{0}}^{n} \mathrm{e}^{\left(t_{n+1}-t_{i+1}\right) A(t)}\left(S_{i}\left(\tau_{i}\right)-\mathrm{e}^{\tau_{i} A(t)}\right) \mathrm{e}^{\left(t_{i}-t_{n_{0}}\right) A(t)}
\end{aligned}
$$

as a consequence, by (3.5), we obtain the estimate

$$
\begin{aligned}
\left\|\Delta_{n_{0}}^{n}\right\|_{X \leftarrow X} \leqslant \| & \Delta_{n_{0}+1}^{n}\left\|_{X \leftarrow X}\right\| S_{n_{0}}\left(\tau_{n_{0}}\right)-\mathrm{e}^{\tau_{n_{0}} A(t)} \|_{X \leftarrow X} \\
& +\sum_{i=n_{0}+1}^{n-1}\left\|\Delta_{i+1}^{n}\right\|_{X \leftarrow X}\left\|S_{i}\left(\tau_{i}\right)-\mathrm{e}^{\tau_{i} A(t)}\right\|_{X \leftarrow D}\left\|\mathrm{e}^{\left(t_{i}-t_{n_{0}}\right) A(t)}\right\|_{D \leftarrow X} \\
& +C\left\|S_{n_{0}}\left(\tau_{n_{0}}\right)-\mathrm{e}^{\tau_{n_{0}} A(t)}\right\|_{X \leftarrow X} \\
& +C \sum_{i=n_{0}+1}^{n}\left\|S_{i}\left(\tau_{i}\right)-\mathrm{e}^{\tau_{i} A(t)}\right\|_{X \leftarrow D}\left\|\mathrm{e}^{\left(t_{i}-t_{n_{0}}\right) A(t)}\right\|_{D \leftarrow X} \\
\leqslant \| & \Delta_{n_{0}+1}^{n}\left\|_{X \leftarrow X}\right\| S_{n_{0}}\left(\tau_{n_{0}}\right)-\mathrm{e}^{\tau_{n_{0}} A(t)} \|_{X \leftarrow X} \\
& +C \sum_{i=n_{0}+1}^{n-1}\left(t_{i}-t_{n_{0}}\right)^{-1}\left\|\Delta_{i+1}^{n}\right\|_{X \leftarrow X}\left\|S_{i}\left(\tau_{i}\right)-\mathrm{e}^{\tau_{i} A(t)}\right\|_{X \leftarrow D} \\
& +C\left\|S_{n_{0}}\left(\tau_{n_{0}}\right)-\mathrm{e}^{\tau_{n_{0}} A(t)}\right\|_{X \leftarrow X} \\
& +C \sum_{i=n_{0}+1}^{n}\left(t_{i}-t_{n_{0}}\right)^{-1}\left\|S_{i}\left(\tau_{i}\right)-\mathrm{e}^{\tau_{i} A(t)}\right\|_{X \leftarrow D} .
\end{aligned}
$$

In a similar manner, for some $i \in\left\{n_{0}, \ldots, n\right\}$, the difference $S_{i}\left(\tau_{i}\right)-\mathrm{e}^{\tau_{i} A(t)}$ is rewritten by means of the telescopic identity

$$
\begin{aligned}
S_{i}\left(\tau_{i}\right)-\mathrm{e}^{\tau_{i} A(t)} & =\prod_{j=1}^{J} \mathrm{e}^{\tau_{i} B_{i j}}-\prod_{j=1}^{J} \mathrm{e}^{b_{j} \tau_{i} A(t)} \\
& =\sum_{\ell=1}^{J} \prod_{j=\ell+1}^{J} \mathrm{e}^{b_{j} \tau_{i} A(t)}\left(\mathrm{e}^{\tau_{i} B_{i \ell}}-\mathrm{e}^{b_{\ell} \tau_{i} A(t)}\right) \prod_{j=1}^{\ell-1} \mathrm{e}^{\tau_{i} B_{i j}}
\end{aligned}
$$


and estimated as follows

$$
\begin{aligned}
\left\|S_{i}\left(\tau_{i}\right)-\mathrm{e}^{\tau_{i} A(t)}\right\| \|_{X \leftarrow D} & \leqslant \sum_{\ell=1}^{J}\left\|\prod_{j=\ell+1}^{J} \mathrm{e}^{b_{j} \tau_{i} A(t)}\right\|_{X \leftarrow X}\left\|\mathrm{e}^{\tau_{i} B_{i \ell}}-\mathrm{e}^{b_{\ell} \tau_{i} A(t)}\right\|_{X \leftarrow D}\left\|\prod_{j=1}^{\ell-1} \mathrm{e}^{\tau_{i} B_{i j}}\right\|_{D \leftarrow D} \\
& \leqslant C \max _{\ell \in\{1, \ldots, J\}}\left\|\mathrm{e}^{\tau_{i} B_{i \ell}}-\mathrm{e}^{b_{\ell} \tau_{i} A(t)}\right\|_{X \leftarrow D}, \\
\left\|S_{i}\left(\tau_{i}\right)-\mathrm{e}^{\tau_{i} A(t)}\right\| \|_{X \leftarrow X} & \leqslant \sum_{\ell=1}^{J}\left\|\prod_{j=\ell+1}^{J} \mathrm{e}^{b_{j} \tau_{i} A(t)}\right\|_{X \leftarrow X}\left\|\mathrm{e}^{\tau_{i} B_{i \ell}}-\mathrm{e}^{b_{\ell} \tau_{i} A(t)}\right\|_{X \leftarrow X}\left\|\prod_{j=1}^{\ell-1} \mathrm{e}^{\tau_{i} B_{i j}}\right\|_{X \leftarrow X} \\
& \leqslant C \max _{\ell \in\{1, \ldots, J\}}\left\|\mathrm{e}^{\tau_{i} B_{i \ell}}-\mathrm{e}^{b_{\ell} \tau_{i} A(t)}\right\|_{X \leftarrow X} .
\end{aligned}
$$

An application of the integral formula of Cauchy yields the representation

$$
\begin{aligned}
\mathrm{e}^{\tau_{i} B_{i \ell}}-\mathrm{e}^{b_{\ell} \tau_{i} A(t)} & =\frac{1}{2 \pi \mathrm{i}} \int_{\Gamma} \mathrm{e}^{\lambda}\left(\left(\lambda I-\tau_{i} B_{i \ell}\right)^{-1}-\left(\lambda I-b_{\ell} \tau_{i} A(t)\right)^{-1}\right) \mathrm{d} \lambda \\
& =\tau_{i} \frac{1}{2 \pi \mathrm{i}} \sum_{k=1}^{K} a_{\ell k} \int_{\Gamma} \mathrm{e}^{\lambda}\left(\lambda I-\tau_{i} B_{i \ell}\right)^{-1}\left(A\left(t_{i}+c_{k} \tau_{i}\right)-A(t)\right)\left(\lambda I-b_{\ell} \tau_{i} A(t)\right)^{-1} \mathrm{~d} \lambda
\end{aligned}
$$

and thus implies the bound

$$
\tau_{i}\left\|\mathrm{e}^{\tau_{i} B_{i \ell}\left(\tau_{i}\right)}-\mathrm{e}^{b_{\ell} \tau_{i} A(t)}\right\|_{X \leftarrow X}+\left\|\mathrm{e}^{\tau_{i} B_{i \ell}\left(\tau_{i}\right)}-\mathrm{e}^{b_{\ell} \tau_{i} A(t)}\right\|_{X \leftarrow D} \leqslant C \tau_{i} \max _{k \in \mathscr{K}}\left|t_{i}+c_{k} \tau_{i}-t\right|^{\vartheta}
$$

see also Hypothesis 1 and Remark 1-2. In regard to (3.6), we set $t=t_{n_{0}}+c_{K} \tau_{n_{0}}$ such that

$$
\tau_{i}\left\|S_{i}\left(\tau_{i}\right)-\mathrm{e}^{\tau_{i} A(t)}\right\|_{X \leftarrow X}+\left\|S_{i}\left(\tau_{i}\right)-\mathrm{e}^{\tau_{i} A(t)}\right\|_{X \leftarrow D} \leqslant C \tau_{i} \max _{k \in \mathscr{K}}\left|t_{i}-t_{n_{0}}+c_{k} \tau_{i}-c_{K} \tau_{n_{0}}\right|^{\vartheta}
$$

this yields the bounds

$$
\begin{gathered}
i=n_{0}: \quad \tau_{n_{0}}\left\|S_{n_{0}}\left(\tau_{n_{0}}\right)-\mathrm{e}^{\tau_{n_{0}} A(t)}\right\|_{X \leftarrow X}+\left\|S_{n_{0}}\left(\tau_{n_{0}}\right)-\mathrm{e}^{\tau_{n_{0}} A(t)}\right\|_{X \leftarrow D} \leqslant C \tau_{n_{0}}^{1+\vartheta}, \\
i \in\left\{n_{0}+1, \ldots, n\right\}: \quad \tau_{i}\left\|S_{i}\left(\tau_{i}\right)-\mathrm{e}^{\tau_{i} A(t)}\right\|_{X \leftarrow X}+\left\|S_{i}\left(\tau_{i}\right)-\mathrm{e}^{\tau_{i} A(t)}\right\|_{X \leftarrow D} \leqslant C \tau_{i}\left(t_{i}-t_{n_{0}}\right)^{\vartheta} .
\end{gathered}
$$

Altogether, by collecting the above bounds and estimating the arising Riemann sums by the corresponding integrals, we obtain

$$
\begin{aligned}
\left\|\Delta_{n_{0}}^{n}\right\|_{X \leftarrow X} \leqslant C & \tau_{n_{0}}^{\vartheta}\left\|\Delta_{n_{0}+1}^{n}\right\|_{X \leftarrow X}+C \sum_{i=n_{0}+1}^{n-1} \tau_{i}\left(t_{i}-t_{n_{0}}\right)^{-1+\vartheta}\left\|\Delta_{i+1}^{n}\right\|_{X \leftarrow X} \\
& +C \tau_{n_{0}}^{\vartheta}+C \sum_{i=n_{0}+1}^{n} \tau_{i}\left(t_{i}-t_{n_{0}}\right)^{-1+\vartheta} \\
\leqslant C & \tau_{n_{0}}^{\vartheta}\left\|\Delta_{n_{0}+1}^{n}\right\|_{X \leftarrow X}+C \sum_{i=n_{0}+1}^{n-1} \tau_{i}\left(t_{i}-t_{n_{0}}\right)^{-1+\vartheta}\left\|\Delta_{i+1}^{n}\right\|_{X \leftarrow X}+C .
\end{aligned}
$$

A Gronwall-type inequality involving a weakly singular kernel, see for instance BRUNNNER, VAN DER HOUWEN (1986), finally proves the stated result. $\quad \square$ 


\subsection{Local error expansion}

Our objective is the derivation of a local error expansion for a high-order CFQM exponential integrator that is appropriate for non-autonomous evolution equations involving time-dependent unbounded linear operators. Compared to alternative approaches for nonstiff differential equations, it is essential to capture the remainder terms and to specify the regularity and compatibility requirements on the problem data and the exact solution. As the treatment of the general case entails certain technicalities, detailed calculations are shifted to the appendix; for the less involved case of a fourth-order CFQM exponential integrator comprising two nodes and two exponentials, the basic expansion steps are also recapitulated there.

LOCAL ERROR We meanwhile fix $n \in\{0,1, \ldots, N-1\}$. For the convenience of the reader, we recall useful abbreviations and a basic consistency condition on the coefficients

$$
\begin{gathered}
A_{n k}=A\left(t_{n}+c_{k} \tau_{n}\right), \quad k \in \mathscr{K}, \\
B_{n j}=\sum_{k=1}^{K} a_{j k} A_{n k}, \quad b_{j}=\sum_{k=1}^{K} a_{j k}, \quad d_{j}=\sum_{\ell=1}^{j} b_{\ell}, \quad j \in \mathscr{J}, \\
d_{J}=\sum_{j=1}^{J} b_{j}=1,
\end{gathered}
$$

see (2.2) and (2.3). The local error of a CFQM exponential integrator is defined by

$$
\delta_{n+1}=u\left(t_{n+1}\right)-S_{n}\left(\tau_{n}\right) u\left(t_{n}\right)=u\left(t_{n+1}\right)-\prod_{j=1}^{J} \mathrm{e}^{\tau_{n} B_{n j}} u\left(t_{n}\right) .
$$

Assuming that the coefficients of the method satisfy the $p$-th nonstiff order conditions, our aim is to deduce a local error expansion which implies $\delta_{n+1}=\mathscr{O}\left(\tau_{n}^{p+1}\right)$.

LINEARISATION AND SOLUTION REPRESENTATION Let $j \in \mathscr{J}$ be such that $b_{j} \neq 0$. In order to attain a local error representation that is appropriate for further stepwise expansions, we rewrite the right-hand side of the evolution equation (1.1) as follows

$$
\begin{gathered}
u^{\prime}(t)=A(t) u(t)=\widetilde{B}_{n j} u(t)+R_{n j}(t), \\
\widetilde{B}_{n j}=\frac{1}{b_{j}} B_{n j}, \quad R_{n j}(t)=\left(A(t)-\widetilde{B}_{n j}\right) u(t), \quad t \in\left[t_{n}, t_{n}+\tau_{n}\right] .
\end{gathered}
$$

The variation-of-constants formula together with a linear integral transformation yields

$$
\begin{aligned}
u\left(t_{n}+d_{j} \tau_{n}\right) & =u\left(t_{n}+d_{j-1} \tau_{n}+b_{j} \tau_{n}\right) \\
& =\mathrm{e}^{\tau_{n} B_{n j}} u\left(t_{n}+d_{j-1} \tau_{n}\right)+b_{j} \int_{0}^{\tau_{n}} \mathrm{e}^{\left(\tau_{n}-\sigma\right) B_{n j}} R_{n j}\left(t_{n}+d_{j-1} \tau_{n}+b_{j} \sigma\right) \mathrm{d} \sigma
\end{aligned}
$$


a repeated application of this relation implies

$$
\begin{aligned}
& u\left(t_{n}+d_{1} \tau_{n}\right)=\mathrm{e}^{\tau_{n} B_{n 1}} u\left(t_{n}\right)+b_{1} \int_{0}^{\tau_{n}} \mathrm{e}^{\left(\tau_{n}-\sigma\right) B_{n 1}} R_{n 1}\left(t_{n}+b_{1} \sigma\right) \mathrm{d} \sigma, \\
& u\left(t_{n}+d_{2} \tau_{n}\right)=\mathrm{e}^{\tau_{n} B_{n 2}} u\left(t_{n}+d_{1} \tau_{n}\right)+b_{2} \int_{0}^{\tau_{n}} \mathrm{e}^{\left(\tau_{n}-\sigma\right) B_{n 2}} R_{n 2}\left(t_{n}+d_{1} \tau_{n}+b_{2} \sigma\right) \mathrm{d} \sigma \\
& =\mathrm{e}^{\tau_{n} B_{n 2}} \mathrm{e}^{\tau_{n} B_{n 1}} u\left(t_{n}\right) \\
& +b_{1} \mathrm{e}^{\tau_{n} B_{n 2}} \int_{0}^{\tau_{n}} \mathrm{e}^{\left(\tau_{n}-\sigma\right) B_{n 1}} R_{n 1}\left(t_{n}+b_{1} \sigma\right) \mathrm{d} \sigma \\
& +b_{2} \int_{0}^{\tau_{n}} \mathrm{e}^{\left(\tau_{n}-\sigma\right) B_{n 2}} R_{n 2}\left(t_{n}+d_{1} \tau_{n}+b_{2} \sigma\right) \mathrm{d} \sigma \\
& u\left(t_{n}+d_{3} \tau_{n}\right)=\mathrm{e}^{\tau_{n} B_{n 3}} u\left(t_{n}+d_{2} \tau_{n}\right)+b_{3} \int_{0}^{\tau_{n}} \mathrm{e}^{\left(\tau_{n}-\sigma\right) B_{n 3}} R_{n 3}\left(t_{n}+d_{2} \tau_{n}+b_{3} \sigma\right) \mathrm{d} \sigma \\
& =\mathrm{e}^{\tau_{n} B_{n 3}} \mathrm{e}^{\tau_{n} B_{n 2}} \mathrm{e}^{\tau_{n} B_{n 1}} u\left(t_{n}\right) \\
& +b_{1} \mathrm{e}^{\tau_{n} B_{n 3}} \mathrm{e}^{\tau_{n} B_{n 2}} \int_{0}^{\tau_{n}} \mathrm{e}^{\left(\tau_{n}-\sigma\right) B_{n 1}} R_{n 1}\left(t_{n}+b_{1} \sigma\right) \mathrm{d} \sigma \\
& +b_{2} \mathrm{e}^{\tau_{n} B_{n 3}} \int_{0}^{\tau_{n}} \mathrm{e}^{\left(\tau_{n}-\sigma\right) B_{n 2}} R_{n 2}\left(t_{n}+d_{1} \tau_{n}+b_{2} \sigma\right) \mathrm{d} \sigma \\
& +b_{3} \int_{0}^{\tau_{n}} \mathrm{e}^{\left(\tau_{n}-\sigma\right) B_{n 3}} R_{n 3}\left(t_{n}+d_{2} \tau_{n}+b_{3} \sigma\right) \mathrm{d} \sigma,
\end{aligned}
$$

and, by induction, this leads to

$$
u\left(t_{n+1}\right)=\prod_{j=1}^{J} \mathrm{e}^{\tau_{n} B_{n j}} u\left(t_{n}\right)+\sum_{j=1}^{J} b_{j}\left(\prod_{i=j+1}^{J} \mathrm{e}^{\tau_{n} B_{n i}}\right) \int_{0}^{\tau_{n}} \mathrm{e}^{\left(\tau_{n}-\sigma\right) B_{n j}} R_{n j}\left(t_{n}+d_{j-1} \tau_{n}+b_{j} \sigma\right) \mathrm{d} \sigma .
$$

LOCAL ERROR REPRESENTATION As a consequence, we obtain the local error representation

$$
\begin{gathered}
\delta_{n+1}=\sum_{j=1}^{J} \sum_{k=1}^{K} a_{j k}\left(\prod_{i=j+1}^{J} \mathrm{e}^{\tau_{n} B_{n i}}\right) \int_{0}^{\tau_{n}} \mathrm{e}^{\left(\tau_{n}-\sigma\right) B_{n j}} g_{n j k}(\sigma) \mathrm{d} \sigma, \\
g_{n j k}(\sigma)=\left(A\left(t_{n}+d_{j-1} \tau_{n}+b_{j} \sigma\right)-A\left(t_{n}+c_{k} \tau_{n}\right)\right) u\left(t_{n}+d_{j-1} \tau_{n}+b_{j} \sigma\right),
\end{gathered}
$$

see also (2.2) and (3.7).

FURTHER EXPANSION Starting from the above integral relation, suitable Taylor series expansions of certain values of $A$ and $u$ lead to a representation of the form

$$
\delta_{n+1}^{(1)}=\sum_{j=1}^{J} \sum_{k=1}^{K} \sum_{\substack{\ell=1 \\ \ell+m \leqslant p-1}}^{p-1} \sum_{\substack{m=0 \\ \ell-2}}^{p-2 k \ell m} \tau_{n}^{\ell+m+1} F_{j k \ell m}\left(B_{n j}, \ldots, B_{n J}\right) A^{(\ell)}\left(t_{n}\right) u^{(m)}\left(t_{n}\right)+\mathscr{O}\left(\tau_{n}^{p+1}, A^{(p)}, u^{(p-1)}\right)
$$


with certain coefficients $c_{j k \ell m}$ and expressions $F_{j k \ell m}$ involving the operators $B_{n i}$ for $j \leqslant i \leqslant J$; detailed calculations are included in the appendix. A further expansion yields the relation

$$
\begin{aligned}
\delta_{n+1}= & \sum_{j=1}^{J} \sum_{k=1}^{K} \sum_{\ell=1}^{p-1} \sum_{\substack{\ell=0 \\
\ell+m \leqslant p-1}}^{p-2} \sum_{\kappa \in \mathscr{K}_{j \ell m}} c_{j k \ell m \kappa} \tau_{n}^{\ell+m+|\kappa|+1} \\
& \times\left(\prod_{i=j}^{J} B_{n i}^{\kappa_{i}}\right) A^{(\ell)}\left(t_{n}\right) u^{(m)}\left(t_{n}\right)+\mathscr{O}\left(\tau_{n}^{p+1}, A^{(p)}, u^{(p-1)}\right), \\
c_{j k \ell m \kappa}= & \frac{\kappa_{j} !}{\ell ! m ! \kappa !} a_{j k}\left(\sum_{\mu=0}^{\ell+m} \frac{(\ell+m) !}{\mu !\left(\ell+m+\kappa_{j}+1-\mu\right) !} b_{j}^{\ell+m-\mu} d_{j-1}^{\mu}-\sum_{v=0}^{m} \frac{m !}{v !\left(m+\kappa_{j}+1-v\right) !} b_{j}^{m-v} c_{k}^{\ell} d_{j-1}^{v}\right),
\end{aligned}
$$

and, in a final step, a suitable expansion of the product involving the operators $B_{n i}$ for $j \leqslant i \leqslant J$ is employed.

LOCAL ERROR EXPANSION Altogether, this yields the local error expansion

$$
\delta_{n+1}=\delta_{n+1}^{(4)}+\mathscr{R}_{n+1},
$$

where the principal term

$$
\begin{aligned}
& \delta_{n+1}^{(4)}=\sum_{j=1}^{J} \sum_{k=1}^{K} \sum_{\ell=1}^{p-1} \sum_{\substack{\ell=0 \\
\ell+m \leqslant p-1}}^{p-2} \sum_{\kappa \in \mathscr{K}_{j \ell m}} \sum_{\lambda \in \mathscr{L}_{j \ell m \kappa}} \frac{\kappa_{j} !}{\ell ! m ! \kappa ! \lambda !} a_{j k} \gamma_{\lambda} \tau_{n}^{\ell+m+|\kappa|+|\lambda|+1} \\
& \times\left(\sum_{\mu=0}^{\ell+m} \frac{(\ell+m) !}{\mu !\left(\ell+m+\kappa_{j}+1-\mu\right) !} b_{j}^{\ell+m-\mu} d_{j-1}^{\mu}\right. \\
& \left.-\sum_{v=0}^{m} \frac{m !}{v !\left(m+\kappa_{j}+1-v\right) !} b_{j}^{m-v} c_{k}^{\ell} d_{j-1}^{v}\right) A^{(\lambda)}\left(t_{n}\right) A^{(\ell)}\left(t_{n}\right) u^{(m)}\left(t_{n}\right)
\end{aligned}
$$

retains the nonstiff order conditions imposed on a $p$-th order CFQM exponential integrator (2.2); more precisely, a (redundant) set of order conditions results from inspecting the expressions associated with $\tau_{n}^{q}$ for exponents $q \in\{2, \ldots, p\}$ and utilising that certain compositions of $A$ and $u$ as well as time derivatives thereof are independent. The remainder comprises terms of the form $A^{(\ell)}(s) u^{(m)}(t)$ with $\ell \in\{0,1, \ldots, p\}$ and $m \in\{0,1, \ldots, p-1\}$.

\subsection{Convergence result}

By means of the stability bound and the local error expansion given in Sections 3.3 and 3.4, we are able to establish the following convergence result for a CFQM exponential integrator of the form (2.2). We point out once more that the local error expansion (3.10) implies that the stiff order conditions coincide with the nonstiff order conditions. A set of ten independent order conditions for nonstiff order five and the corresponding seven conditions for time-symmetric schemes of order six are stated in our related work BLANES, CASAS, ThalHAMmer (2016), see also references given therein. For notational simplicity, we formulate the global error estimate for the maximum time stepsize

$$
\tau=\max \left\{\tau_{n}: n \in\{0,1, \ldots, N-1\}\right\} .
$$


On the basis of the elementary evolution equation introduced in Section 3.2, the regularity and compatibility requirements on the problem data and the exact solution are discussed below.

Theorem 2 Assume that the operator family $(A(t))_{t \in\left[t_{0}, T\right]}$ defining the non-autonomous linear evolution equation (1.1) satisfies Hypothesis 1; suppose further that the coefficients of the considered CFQM exponential integrator fulfill the relations in (2.3) and the nonstiff $p$-th order conditions. Provided that compositions of the form $A^{(\ell)}(s) u^{(m)}(t)$ with $\ell \in\{0,1, \ldots, p\}$ and $m \in\{0,1, \ldots, p-1\}$ remain bounded for $s, t \in[0, T]$, the global error estimate

$$
\left\|u_{n}-u\left(t_{n}\right)\right\|_{X} \leqslant C\left(\left\|u_{0}-u\left(t_{0}\right)\right\|_{X}+\tau^{p}\right), \quad n \in\{0,1, \ldots, N\},
$$

is valid with a constant $C>0$ that does not dependent on $n$ and $\tau$.

PROOF. As standard, we employ a telescopic identity to relate the global error to the local errors

$$
\begin{gathered}
u_{n}-u\left(t_{n}\right)=\left(\prod_{\ell=0}^{n-1} S_{\ell}\left(\tau_{\ell}\right)\right)\left(u_{0}-u\left(t_{0}\right)\right)-\sum_{m=0}^{n-1}\left(\prod_{\ell=m+1}^{n-1} S_{\ell}\left(\tau_{\ell}\right)\right) \delta_{m+1}, \\
\delta_{m+1}=u\left(t_{m+1}\right)-\left(S_{m}\left(\tau_{m}\right) u\left(t_{m}\right) .\right.
\end{gathered}
$$

By means of Theorem 1, the estimate

$$
\left\|u_{n}-u\left(t_{n}\right)\right\|_{X} \leqslant C\left\|u_{0}-u\left(t_{0}\right)\right\|_{X}+C \sum_{m=0}^{n-1}\left\|\delta_{m+1}\right\|_{X}
$$

follows. Under the given assumptions on the operator family and the exact solution, the local error expansion (3.10) is well-defined; in particular, the remainder is bounded in the underlying Banach space

$$
\left\|\mathscr{R}_{m+1}\right\|_{X} \leqslant C \tau_{m}^{p+1} .
$$

Provided that the CFQM exponential integrator (2.2) fulfills the nonstiff $p$-th order conditions, the principal error term in (3.10) vanishes, and we obtain

$$
\delta_{m+1}^{(4)}=0, \quad\left\|\delta_{m+1}\right\|_{X}=\left\|\mathscr{R}_{m+1}\right\|_{X} \leqslant C \tau_{m}^{p+1} .
$$

As a consequence, this implies the stated global error bound.

Remark 3 We note that any CFQM exponential integrator of order five or higher found in the literature involves merely real coefficients and does not fulfill the positivity condition in (2.3); as a consequence, the above result does not apply to it.

ELEMENTARY EVOLUTION EQUATION In order to substantiate the implications of Theorem 2, we reconsider the elementary evolution equation (3.4), choosing again the space of continuous functions as underlying function space. Provided that the coefficient functions are sufficiently often continuously differentiable, time derivatives of the defining operator again correspond to second-order differential operators; for instance, the first time derivative is given by

$$
A^{\prime}(t): D \longrightarrow X: w \longmapsto\left[x \mapsto \partial_{t} \alpha_{2}(x, t) \partial_{x x} w(x)+\partial_{t} \alpha_{1}(x, t) \partial_{x} w(x)+\partial_{t} \alpha_{0}(x, t) w(x)\right], \quad t \in\left[t_{0}, T\right] .
$$


Straighforward calculations such as

$$
\begin{aligned}
u^{\prime}(t) & =A(t) u(t), \\
u^{\prime \prime}(t) & =\left(A^{\prime}(t)+A(t) A(t)\right) u(t), \\
u^{\prime \prime \prime}(t) & =\left(A^{\prime \prime}(t)+2 A^{\prime}(t) A(t)+A(t) A^{\prime}(t)+A(t) A(t) A(t)\right) u(t),
\end{aligned}
$$

show that the $k$-th time derivative of the solution involves the $k$-fold product $(A(t))^{k}$ for $k \in \mathbb{N}$. The regularity requirements of Theorem 2 , reflected for instance in

$$
A^{(p)}(t) u^{(p-1)}(t) \in \mathscr{C}(\Omega, \mathbb{R}),
$$

hence correspond to the condition

$$
u(t) \in \mathscr{C}^{2 p}(\Omega, \mathbb{R}), \quad t \in\left[t_{0}, T\right] .
$$

Whenever the solution is subject to boundary conditions, additional restrictions apply; for instance, in the case of homogeneous Dirichlet boundary conditions, the condition $u^{(p-1)}(t) \in D$ in particular involves the compatibility requirement

$$
\left.\partial_{x}^{2(p-1)} u(x, t)\right|_{x \in \partial \Omega}=0, \quad t \in\left[t_{0}, T\right]
$$

ORDER REDUCTIONS In situations, where it is unnatural to assume that certain compositions of the defining operators and the exact solution satisfy the imposed boundary conditions, order reductions are encountered. These order reductions are explained by the fact that the relation $(A(t))^{k-\kappa} u(t) \in X$ holds for certain exponents $k \in \mathbb{N}$ and $\kappa \in(0,1)$, but $(A(t))^{k} u(t) \notin X$. The smoothing property of an analytic semigroup, reflected in relation (3.3), then permits to use estimates such as

$$
t^{\kappa}\left\|(A(t))^{\kappa} \mathrm{e}^{t A(t)}(A(t))^{k-\kappa} u(t)\right\|_{X} \leqslant C\left\|(A(t))^{k-\kappa} u(t)\right\|_{X}, \quad k \in \mathbb{N}, \quad \kappa \in(0,1),
$$

in order to establish global error estimates involving fractional powers of the time increments. For a detailed error analysis of a fourth-order CFQM exponential integrator and the explanation of significant order reductions, we refer to THALHAMMER (2006); a numerical counterexample for an evolution equation subject to homogeneous Dirichlet boundary conditions is also given there.

FULL DISCRETISATION ERROR Our convergence result for the time-discrete solution is also a basic ingredient for the derivation of an estimate for the full discretisation error. We sketch the approach for the exponential midpoint rule applied with constant time stepsize $\tau>0$ and exact initial value

$$
u_{n}=\prod_{\ell=0}^{n-1} S_{\ell}(\tau) u\left(t_{0}\right)=\prod_{\ell=0}^{n-1} \mathrm{e}^{\tau A\left(t_{\ell}+\frac{1}{2} \tau\right)} u\left(t_{0}\right), \quad n \in\{1, \ldots, N\},
$$

see also (1.4); the fully discrete solution values are obtained by a composition of the form

$$
u_{n M}=\prod_{\ell=0}^{n-1} \widetilde{S}_{\ell M}(\tau) u\left(t_{0}\right), \quad n \in\{1, \ldots, N\},
$$

where the index $M$ reflects the quality of the spatial approximation. For instance, for central finite differences, the number $M$ corresponds to the number of spatial grid points. Together with a suitable 
interpolation, this procedure defines an element in the underlying function space (and not just a vector comprising real numbers). The triangle inequality implies that the full discretisation error is bounded by the time discretisation error, see Theorem 2 , and by the error of the additional space discretisation

$$
\left\|u_{n M}-u\left(t_{n}\right)\right\|_{X} \leqslant C \tau^{p}+\left\|u_{n M}-u_{n}\right\|_{X}, \quad n \in\{1, \ldots, N\} .
$$

Employing a telescopic identity, the difference $u_{n M}-u_{n}$ takes the form

$$
\left(\prod_{\ell=0}^{n-1} \widetilde{S}_{\ell M}(\tau)-\prod_{\ell=0}^{n-1} S_{\ell}(\tau)\right) u\left(t_{0}\right)=\sum_{\ell=0}^{n-1}\left(\prod_{m=\ell+1}^{n-1} \widetilde{S}_{m M}(\tau)\right)\left(\widetilde{S}_{\ell M}(\tau)-S_{\ell}(\tau)\right)\left(\prod_{m=0}^{\ell-1} S_{m}(\tau)\right) u\left(t_{0}\right)
$$

Thus, the crucial point is to estimate products of $\widetilde{S}_{\ell M}(\tau)$ and the defect $\widetilde{S}_{\ell M}(\tau)-S_{\ell}(\tau)$. Under suitable regularity requirements on the data of the problem, a global error bound of the form

$$
\left\|u_{n M}-u\left(t_{n}\right)\right\|_{X} \leqslant C\left(\tau^{p}+M^{-q}\right), \quad n \in\{1, \ldots, N\},
$$

with some exponent $q>0$ is expected, see also THALHAMMER (2012) and references given therein.

\section{Numerical examples}

In this section, we present numerical comparisons for a test equation in a single space dimension. The definition of the underlying nonlinear equation is somewhat inspired by models arising in pattern formation such as the Kuramoto-Sivashinsky equation and the Gierer-Meinhardt equations, but with a nonlinear diffusion term; for this type of problems, it is also natural to consider periodic boundary conditions. Our test equation is rather elementary from a computational point of view and has been chosen in this way for two reasons. First, in order to enable a comparison with other high-order CFQM exponential integrators given in the literature, a sufficiently low degree of freedom is required; otherwise, the poor stability behaviour of CFQM exponential integrators involving merely real coefficients would entirely spoil the numerical result (not a number). Second, our purpose here is to confirm the favourable stability behaviour of schemes involving complex coefficients and our theoretical global error estimate, rather than exhaustively analysing the efficiency of our novel schemes on realistic problems.

Simulations for relevant applications are found in Alvermann, Fehske, LitTlewood (2012); for a more detailed assessment of our novel schemes when applied to a dissipative quantum system of higher dimension, we refer to BlANES, CASAS, THALHAMMER (2016).

TEST EQuation Let $\Omega=[0,1]$. In regard to (1.2) and Section 3.2, we consider the nonlinear diffusion-advection-reaction equation

$$
\begin{gathered}
\partial_{t} U(x, t)=f_{2}(U(x, t)) \partial_{x x} U(x, t)+f_{1}(U(x, t)) \partial_{x} U(x, t)+f_{0}(U(x, t)) \\
+g(x, t), \quad(x, t) \in \Omega \times[0, T],
\end{gathered}
$$

defined by the scalar functions

$$
f_{2}(w)=\frac{1}{10}\left(\cos (w)+\frac{11}{10}\right), \quad f_{1}(w)=\frac{1}{10} w, \quad f_{0}(w)=w\left(w-\frac{1}{2}\right) ;
$$

the inhomogeneity is chosen such that the exact solution is equal to

$$
U(x, t)=\mathrm{e}^{-t} \sin (2 \pi x) .
$$



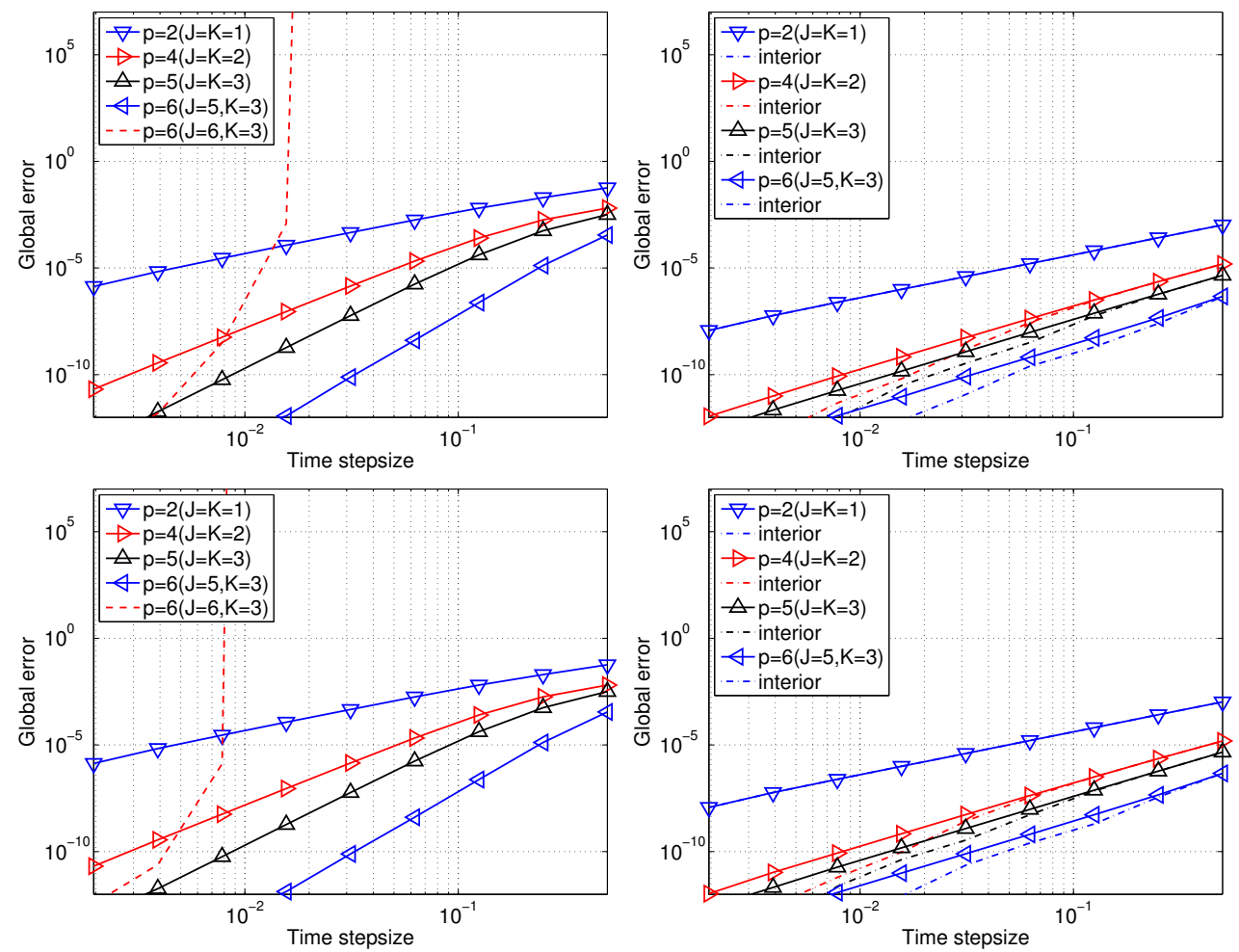

FIG. 1. Time integration of test equation (4.1) by different CFQM exponential integrators of nonstiff orders $p=2,4,5,6$. Left: Periodic boundary conditions. Global errors at time $T=1$ versus the chosen time stepsizes. Right: Homogeneous Dirichlet boundary conditions. Global errors at time $T=1$, measured on the entire spatial domain and in the interior, respectively, versus the chosen time stepsizes. Numerical results obtained for $M=100$ (first row) and $M=120$ (second row) grid points.

The associated variational equation

$$
\partial_{t} u(x, t)=\alpha_{2}(x, t) \partial_{x x} u(x, t)+\alpha_{1}(x, t) \partial_{x} u(x, t)+\alpha_{0}(x, t) u(x, t), \quad(x, t) \in \Omega \times[0, T],
$$

involves the space-time-dependent coefficient functions

$$
\begin{gathered}
\alpha_{2}(x, t)=f_{2}(U(x, t)), \quad \alpha_{1}(x, t)=f_{1}(U(x, t)), \\
\alpha_{0}(x, t)=f_{2}^{\prime}(U(x, t)) \partial_{x x} U(x, t)+f_{1}^{\prime}(U(x, t)) \partial_{x} U(x, t)+f_{0}^{\prime}(U(x, t)),
\end{gathered}
$$

where all values of the leading coefficient are positive; we in particular consider the initial state

$$
u(x, 0)=(\sin (2 \pi x))^{2}, \quad x \in \Omega .
$$

NUMERICAL RESUlTS For the space discretisation, we choose $M=100$ equidistant grid points and apply standard symmetric finite differences. The time integration is performed by different CFQM ex- 
ponential integrators with time stepsizes $2^{-\ell}$ for $\ell \in\{1, \ldots, 10\}$; the numerical approximation obtained for the finest time stepsize serves as reference solution.

In Figure 1, we display the global errors at time $T=1$, obtained for the CFQM exponential integrators of nonstiff orders $p=2,4,5,6$ introduced in Section 2; the slopes of the lines reflect the orders of convergence. In accordance with Theorem 2, the nonstiff orders are retained for smaller time stepsizes.

The numerical comparisons also confirm the poor stability behaviour of CFQM exponential integrators that do not verify condition (2.3c). Indeed, for the sixth-order scheme with real coefficients a satisfactory result is only obtained for sufficiently small time stepsizes; for larger values it fails (not a number). This unstable behaviour changes for the worse when the spatial grid is refined, see Figure 1.

ORDER REDUCTIONS In situations, where compositions of the involved operators applied to the exact solution values do not satisfy the imposed boundary conditions, an order reduction is expected, see THALHAMmer (2006). In order to illustrate this error behaviour, we consider (4.1) with

$$
U(x, t)=\mathrm{e}^{-t} x(1-x), \quad u(x, 0)=10 x(1-x),
$$

and impose homogeneous Dirichlet boundary conditions. We determine the global temporal orders at time $T=1$. In addition, we measure the global errors in the interior of the domain; that is, we only consider the 80 interior grid points and neglect the contributions of the grid points nearby the boundaries.

The obtained results, displayed in Figure 1, show order reductions of the fourth-, fifth- and sixthorder schemes to third-order, when the error is measured in the maximum norm, whereas higher convergence rates are obtained in the interior of the spatial domain.

EFFECT OF SPACE DISCRETISATION For comparison, we include the corresponding results obtained for $M=120$ grid points in Figure 1; due to the fact that the size of the errors are retained for the stable schemes, we conclude that the spatial error is negligible and that the numerical results are independent of the considered space discretisation method.

\section{Acknowledgement}

We thank the associate editor and the anonymous reviewers for their valuable comments. We acknowledge financial support by the Universität der Bundeswehr München and by the Austrian Science Fund (FWF) under project P21620-N13. The work of SB and FC has been additionally supported by Ministerio de Economía y Competitividad (Spain) through projects MTM2013-46553-C3 and MTM201677660-P (AEI/FEDER, UE).

\section{A. Further expansion of the local error}

This section is devoted to a detailed derivation of the local error expansion (3.10), starting from (3.8).

AUXILIARY NOTATION AND RESULTS In a first expansion step, we employ Taylor series expansions of the integrand; that is, we utilise the formula

$$
\begin{gathered}
f(t+s)=\sum_{i=0}^{m} \frac{1}{i !} s^{i} f^{(i)}(t)+R\left(s^{m+1}, f^{(m+1)}\right), \\
R\left(s^{m+1}, f^{(m+1)}\right)=\frac{1}{m !} s^{m+1} \int_{0}^{1}(1-\sigma)^{m} f^{(m+1)}(t+\sigma s) \mathrm{d} \sigma .
\end{gathered}
$$


In order to obtain a compact representation of the resulting integrals and in view of a further expansion, it is convenient to introduce linear operators which are closely related to the analytic semigroup generated by a sectorial operator. For $t \in\left[t_{0}, T\right], \sigma \geqslant 0$, and $m \in \mathbb{N}$, we set

$$
\varphi_{m}(\sigma A(t))= \begin{cases}\mathrm{e}^{\sigma A(t)}, & m=0, \\ \frac{1}{(m-1) !} \sigma^{-m} \int_{0}^{\sigma} \widetilde{\sigma}^{m-1} \mathrm{e}^{(\sigma-\widetilde{\sigma}) A(t)} \mathrm{d} \widetilde{\sigma}, & m \geqslant 1 ;\end{cases}
$$

referred as $\varphi$-functions. Evaluation at zero is understood as right-sided limit $\sigma \rightarrow 0_{+}$such that $\varphi_{m}(0)=\frac{1}{m !} I$ for any $m \in \mathbb{N}$. The corresponding result for the analytic semigroup $\left(\mathrm{e}^{\sigma A(t)}\right)_{\sigma \geqslant 0}$ ensures that $\varphi_{m}(\sigma A(t)): X_{\mu} \rightarrow X_{\mu}$ remains bounded for exponents $\mu \in[0,1]$ and $\sigma \in\left[0, T_{\sigma}\right]$, see (3.3). By means of the recurrence relation

$$
\varphi_{m}(\sigma A(t))=\frac{1}{m !} I+\sigma A(t) \varphi_{m+1}(\sigma A(t)), \quad m \in \mathbb{N},
$$

we obtain the expansion

$$
\varphi_{m}(\sigma A(t))=\sum_{\kappa=0}^{M-1} \frac{1}{(m+\kappa) !}(\sigma A(t))^{\kappa}+(\sigma A(t))^{M} \varphi_{m+M}(\sigma A(t)), \quad m \in \mathbb{N}, \quad M \in \mathbb{N}_{\geqslant 2} .
$$

TAYlor SERIES EXPANSIONS AND INTEgRation Let $(j, k) \in \mathscr{J} \times \mathscr{K}$. In order to expand the local error (3.8) with respect to the time increment, we employ the Taylor series expansions

$$
\begin{gathered}
A\left(t_{n}+d_{j-1} \tau_{n}+b_{j} \sigma\right)-A\left(t_{n}+c_{k} \tau_{n}\right)=\sum_{\ell=1}^{q} \frac{1}{\ell !}\left(\left(d_{j-1} \tau_{n}+b_{j} \sigma\right)^{\ell}-\left(c_{k} \tau_{n}\right)^{\ell}\right) A^{(\ell)}\left(t_{n}\right) \\
+R\left(\left(d_{j-1} \tau_{n}+b_{j} \sigma\right)^{q+1}, A^{(q+1)}\right)-R\left(\left(c_{k} \tau_{n}\right)^{q+1}, A^{(q+1)}\right) \\
u\left(t_{n}+d_{j-1} \tau_{n}+b_{j} \sigma\right)=\sum_{m=0}^{r} \frac{1}{m !}\left(d_{j-1} \tau_{n}+b_{j} \sigma\right)^{m} u^{(m)}\left(t_{n}\right) \\
+R\left(\left(d_{j-1} \tau_{n}+b_{j} \sigma\right)^{r+1}, u^{(r+1)}\right)
\end{gathered}
$$

see (A.1); clearly, the term involving $\ell=0$ vanishes. An elementary calculation yields

$$
\begin{aligned}
g_{n j k}(\sigma)=\sum_{\ell=1}^{q} & \sum_{m=0}^{r} \frac{1}{\ell ! m !}\left(\left(d_{j-1} \tau_{n}+b_{j} \sigma\right)^{\ell}-\left(c_{k} \tau_{n}\right)^{\ell}\right)\left(d_{j-1} \tau_{n}+b_{j} \sigma\right)^{m} A^{(\ell)}\left(t_{n}\right) u^{(m)}\left(t_{n}\right)+R_{j k}(\sigma) \\
R_{j k}(\sigma)=R & \left(\left(d_{j-1} \tau_{n}+b_{j} \sigma\right)^{q+1}, A^{(q+1)}\right) \sum_{m=0}^{r} \frac{1}{m !}\left(d_{j-1} \tau_{n}+b_{j} \sigma\right)^{m} u^{(m)}\left(t_{n}\right) \\
& -R\left(\left(c_{k} \tau_{n}\right)^{q+1}, A^{(q+1)}\right) \sum_{m=0}^{r} \frac{1}{m !}\left(d_{j-1} \tau_{n}+b_{j} \sigma\right)^{m} u^{(m)}\left(t_{n}\right) \\
& +\sum_{\ell=1}^{q} \frac{1}{\ell !}\left(\left(d_{j-1} \tau_{n}+b_{j} \sigma\right)^{\ell}-\left(c_{k} \tau_{n}\right)^{\ell}\right) A^{(\ell)}\left(t_{n}\right) R\left(\left(d_{j-1} \tau_{n}+b_{j} \sigma\right)^{r+1}, u^{(r+1)}\right) \\
& +R\left(\left(d_{j-1} \tau_{n}+b_{j} \sigma\right)^{q+1}, A^{(q+1)}\right) R\left(\left(d_{j-1} \tau_{n}+b_{j} \sigma\right)^{r+1}, u^{(r+1)}\right) \\
& -R\left(\left(c_{k} \tau_{n}\right)^{q+1}, A^{(q+1)}\right) R\left(\left(d_{j-1} \tau_{n}+b_{j} \sigma\right)^{r+1}, u^{(r+1)}\right)
\end{aligned}
$$


Inserting the above representation into (3.8) implies

$$
\begin{aligned}
\delta_{n+1}=\sum_{j=1}^{J} \sum_{k=1}^{K} & \sum_{\ell=1}^{q} \sum_{m=0}^{r} \frac{1}{\ell ! m !} a_{j k}\left(\prod_{i=j+1}^{J} \mathrm{e}^{\tau_{n} B_{n i}}\right) \\
& \times \int_{0}^{\tau_{n}} \mathrm{e}^{\left(\tau_{n}-\sigma\right) B_{n j}}\left(\left(d_{j-1} \tau_{n}+b_{j} \sigma\right)^{\ell}-\left(c_{k} \tau_{n}\right)^{\ell}\right)\left(d_{j-1} \tau_{n}+b_{j} \sigma\right)^{m} \mathrm{~d} \sigma A^{(\ell)}\left(t_{n}\right) u^{(m)}\left(t_{n}\right) \\
& +\sum_{j=1}^{J} \sum_{k=1}^{K} a_{j k}\left(\prod_{i=j+1}^{J} \mathrm{e}^{\tau_{n} B_{n i}}\right) \int_{0}^{\tau_{n}} \mathrm{e}^{\left(\tau_{n}-\sigma\right) B_{n j}} R_{j k}(\sigma) \mathrm{d} \sigma .
\end{aligned}
$$

The integrals arising in the principal local error term are rewritten by means of the binomial series and the $\varphi$-functions, which leads to

$$
\begin{aligned}
& \int_{0}^{\tau_{n}} \mathrm{e}^{\left(\tau_{n}-\sigma\right) B_{n j}}\left(\left(d_{j-1} \tau_{n}+b_{j} \sigma\right)^{\ell}-\left(c_{k} \tau_{n}\right)^{\ell}\right)\left(d_{j-1} \tau_{n}+b_{j} \sigma\right)^{m} \mathrm{~d} \sigma \\
& =\sum_{\mu=0}^{\ell+m}\left(\begin{array}{c}
\ell+m \\
\mu
\end{array}\right) b_{j}^{\ell+m-\mu} d_{j-1}^{\mu} \tau_{n}^{\mu} \int_{0}^{\tau_{n}} \mathrm{e}^{\left(\tau_{n}-\sigma\right) B_{n j}} \sigma^{\ell+m-\mu} \mathrm{d} \sigma \\
& \quad-\sum_{v=0}^{m}\left(\begin{array}{c}
m \\
v
\end{array}\right) b_{j}^{m-v} c_{k}^{\ell} d_{j-1}^{v} \tau_{n}^{\ell+v} \int_{0}^{\tau_{n}} \mathrm{e}^{\left(\tau_{n}-\sigma\right) B_{n j}} \sigma^{m-v} \mathrm{~d} \sigma \\
& =\tau_{n}^{\ell+m+1}\left(\sum_{\mu=0}^{\ell+m} \frac{(\ell+m) !}{\mu !} b_{j}^{\ell+m-\mu} d_{j-1}^{\mu} \varphi_{\ell+m+1-\mu}\left(\tau_{n} B_{n j}\right)-\sum_{\nu=0}^{m} \frac{m !}{v !} b_{j}^{m-v} c_{k}^{\ell} d_{j-1}^{v} \varphi_{m+1-v}\left(\tau_{n} B_{n j}\right)\right) .
\end{aligned}
$$

As a consequence, choosing $q=p-1$ and $r=q-1=p-2$, where $p$ denotes the nonstiff order of (2.2), we obtain the local error representation

$$
\begin{gathered}
\delta_{n+1}=\delta_{n+1}^{(1)}+\mathscr{R}_{n+1}^{(1)} \\
\delta_{n+1}^{(1)}=\sum_{j=1}^{J} \sum_{k=1}^{K} \sum_{\ell=1}^{p-1} \sum_{m=0}^{p-2} \frac{1}{\ell ! m !} a_{j k} \tau_{n}^{\ell+m+1}\left(\prod_{i=j+1}^{J} \mathrm{e}^{\tau_{n} B_{n i}}\right) \\
\times\left(\sum_{\mu=0}^{\ell+m \leqslant p-1} \frac{(\ell+m) !}{\mu !} b_{j}^{\ell+m-\mu} d_{j-1}^{\mu} \varphi_{\ell+m+1-\mu}\left(\tau_{n} B_{n j}\right)\right. \\
\left.-\sum_{v=0}^{m} \frac{m !}{v !} b_{j}^{m-v} c_{k}^{\ell} d_{j-1}^{v} \varphi_{m+1-v}\left(\tau_{n} B_{n j}\right)\right) A^{(\ell)}\left(t_{n}\right) u^{(m)}\left(t_{n}\right) ;
\end{gathered}
$$


in order to indicate the dependence of the remainder

$$
\begin{aligned}
\mathscr{R}_{n+1}^{(1)}=\sum_{j=1}^{J} & \sum_{k=1}^{K} \sum_{\ell=1}^{p-1} \sum_{m=0}^{p-2} \frac{1}{\ell ! m !} a_{j k} \tau_{n}^{\ell+m+1}\left(\prod_{i=j+1}^{J} \mathrm{e}^{\tau_{n} B_{n i}}\right) \\
& \times \sum_{\mu=0}^{\ell+m} \frac{(\ell+m) !}{\mu !} b_{j}^{\ell+m-\mu} d_{j-1}^{\mu} \varphi_{\ell+m+1-\mu}\left(\tau_{n} B_{n j}\right) \\
& \left.-\sum_{v=0}^{m} \frac{m !}{v !} b_{j}^{m-v} c_{k}^{\ell} d_{j-1}^{v} \varphi_{m+1-v}\left(\tau_{n} B_{n j}\right)\right) A^{(\ell)}\left(t_{n}\right) u^{(m)}\left(t_{n}\right) \\
& +\sum_{j=1}^{J} \sum_{k=1}^{K} a_{j k}\left(\prod_{i=j+1}^{J} \mathrm{e}^{\tau_{n} B_{n i}}\right) \int_{0}^{\tau_{n}} \mathrm{e}^{\left(\tau_{n}-\sigma\right) B_{n j}} R_{j k}(\sigma) \mathrm{d} \sigma \\
R_{j k}(\sigma)=R & \left.\left(d_{j-1} \tau_{n}+b_{j} \sigma\right)^{p}, A^{(p)}\right) \sum_{m=0}^{p-2} \frac{1}{m !}\left(d_{j-1} \tau_{n}+b_{j} \sigma\right)^{m} u^{(m)}\left(t_{n}\right) \\
& -R\left(\left(c_{k} \tau_{n}\right)^{p}, A^{(p)}\right) \sum_{m=0}^{p-2} \frac{1}{m !}\left(d_{j-1} \tau_{n}+b_{j} \sigma\right)^{m} u^{(m)}\left(t_{n}\right) \\
& +\sum_{\ell=1}^{p-1} \frac{1}{\ell !}\left(\left(d_{j-1} \tau_{n}+b_{j} \sigma\right)^{\ell}-\left(c_{k} \tau_{n}\right)^{\ell}\right) A^{(\ell)}\left(t_{n}\right) R\left(\left(d_{j-1} \tau_{n}+b_{j} \sigma\right)^{p-1}, u^{(p-1)}\right) \\
+ & R\left(\left(d_{j-1} \tau_{n}+b_{j} \sigma\right)^{p}, A^{(p)}\right) R\left(\left(d_{j-1} \tau_{n}+b_{j} \sigma\right)^{p-1}, u^{(p-1)}\right) \\
& -R\left(\left(c_{k} \tau_{n}\right)^{p}, A^{(p)}\right) R\left(\left(d_{j-1} \tau_{n}+b_{j} \sigma\right)^{p-1}, u^{(p-1)}\right)
\end{aligned}
$$

on the time increment and the highest time derivatives, we employ the short notation

$$
\mathscr{R}_{n+1}^{(1)}=\mathscr{O}\left(\tau_{n}^{p+1}, A^{(p)}, u^{(p-1)}\right)
$$

EXPANSION OF EXPONENTIAL FUNCTIONS It remains to suitably expand the principal term in the local error representation (A.3). For this purpose, we apply the following relations for the $\varphi$-functions

$$
\begin{aligned}
\varphi_{\ell+m+1-\mu}\left(\tau_{n} B_{n j}\right) & =\sum_{\kappa=0}^{p-\ell-m-1} \frac{1}{(\ell+m+\kappa+1-\mu) !}\left(\tau_{n} B_{n j}\right)^{\kappa}+\left(\tau_{n} B_{n j}\right)^{p-\ell-m} \varphi_{p+1-\mu}\left(\tau_{n} B_{n j}\right), \\
\varphi_{m+1-v}\left(\tau_{n} B_{n j}\right) & =\sum_{\kappa=0}^{p-\ell-m-1} \frac{1}{(m+\kappa+1-v) !}\left(\tau_{n} B_{n j}\right)^{\kappa}+\left(\tau_{n} B_{n j}\right)^{p-\ell-m} \varphi_{p+1-\ell-v}\left(\tau_{n} B_{n j}\right),
\end{aligned}
$$

see (A.2). This leads to the local error expansion

$$
\delta_{n+1}=\delta_{n+1}^{(2)}+\mathscr{R}_{n+1}^{(1)}+\mathscr{R}_{n+1}^{(2)}
$$




$$
\begin{aligned}
\delta_{n+1}^{(2)}=\sum_{j=1}^{J} & \sum_{k=1}^{K} \sum_{\substack{\ell=1 \\
\ell+m \leqslant p-1}}^{p-1} \sum_{m=0}^{p-2} \sum_{\kappa=0}^{p-\ell-m-1} \frac{1}{\ell ! m !} a_{j k} \tau_{n}^{\ell+m+\kappa+1} \\
& \times\left(\sum_{\mu=0}^{\ell+m} \frac{(\ell+m) !}{\mu !(\ell+m+\kappa+1-\mu) !} b_{j}^{\ell+m-\mu} d_{j-1}^{\mu}-\sum_{v=0}^{m} \frac{m !}{v !(m+\kappa+1-v) !} b_{j}^{m-v} c_{k}^{\ell} d_{j-1}^{v}\right) \\
& \times\left(\prod_{i=j+1}^{J} \mathrm{e}^{\tau_{n} B_{n i}}\right) B_{n j}^{\kappa} A^{(\ell)}\left(t_{n}\right) u^{(m)}\left(t_{n}\right),
\end{aligned}
$$

where the remainder is given by $(\mathrm{A} .3 \mathrm{c})$ and

$$
\begin{aligned}
\mathscr{R}_{n+1}^{(2)}=\tau_{n}^{p+1} & \sum_{j=1}^{J} \sum_{k=1}^{K} \sum_{\substack{\ell=1 \\
\ell+m \leqslant p-1}}^{p-1} \sum_{m=0}^{p-2} \frac{1}{\ell ! m !} a_{j k}\left(\prod_{i=j+1}^{J} \mathrm{e}^{\tau_{n} B_{n i}}\right) \\
& \times\left(\sum_{\mu=0}^{\ell+m} \frac{(\ell+m) !}{\mu !} b_{j}^{\ell+m-\mu} d_{j-1}^{\mu} \varphi_{p+1-\mu}\left(\tau_{n} B_{n j}\right)\right. \\
& \left.\quad-\sum_{v=0}^{m} \frac{m !}{v !} b_{j}^{m-v} c_{k}^{\ell} d_{j-1}^{v} \varphi_{p+1-\ell-v}\left(\tau_{n} B_{n j}\right)\right) B_{n j}^{p-\ell-m} A^{(\ell)}\left(t_{n}\right) u^{(m)}\left(t_{n}\right)
\end{aligned}
$$

as before, we indicate the highest time-derivatives and write

$$
\mathscr{R}_{n+1}^{(1)}+\mathscr{R}_{n+1}^{(2)}=\mathscr{O}\left(\tau_{n}^{p+1}, A^{(p)}, u^{(p-1)}\right)
$$

for short. In a further expansion step, we repeatedly employ the recurrence relation

$$
\mathrm{e}^{\tau_{n} B_{n i}}=\varphi_{0}\left(\tau_{n} B_{n i}\right)=\sum_{\lambda_{i}=0}^{\Lambda_{i}-1} \frac{1}{\lambda_{i} !} \tau_{n}^{\lambda_{i}} B_{n i}^{\lambda_{i}}+\tau_{n}^{\Lambda_{i}} B_{n i}^{\Lambda_{i}} \varphi_{\Lambda_{i}}\left(\tau_{n} B_{n i}\right)
$$

Setting $\Lambda_{J}=p-\ell-m-\kappa$ as well as $\Lambda_{J-1}=\Lambda_{J}-\lambda_{J}$, we obtain

$$
\begin{aligned}
\mathrm{e}^{\tau_{n} B_{n J}} \mathrm{e}^{\tau_{n} B_{n, J-1}=} & \sum_{\lambda_{J}=0}^{\Lambda_{J}-1} \frac{1}{\lambda_{J} !} \tau_{n}^{\lambda_{J}} B_{n J}^{\lambda_{J}} \mathrm{e}^{\tau_{n} B_{n, J-1}}+\tau_{n}^{\Lambda_{J}} B_{n J}^{\Lambda_{J}} \varphi_{\Lambda_{J}}\left(\tau_{n} B_{n J}\right) \mathrm{e}^{\tau_{n} B_{n, J-1}} \\
= & \sum_{\lambda_{J}=0}^{\Lambda_{J}-1} \sum_{\lambda_{J-1}=0}^{\Lambda_{J-1}-1} \frac{1}{\lambda_{J-1} ! \lambda_{J} !} \tau_{n}^{\lambda_{J-1}+\lambda_{J}} B_{n J}^{\lambda_{J}} B_{n, J-1}^{\lambda_{J-1}} \\
& +\sum_{\lambda_{J}=0}^{\Lambda_{J}-1} \frac{1}{\lambda_{J} !} \tau_{n}^{\lambda_{J}+\Lambda_{J-1}} B_{n J}^{\lambda_{J}} B_{n, J-1}^{\Lambda_{J-1}} \varphi_{\Lambda_{J-1}}\left(\tau_{n} B_{n, J-1}\right) \\
= & \quad+\tau_{n}^{\Lambda_{J}} B_{n J}^{\Lambda_{J}} \varphi_{\Lambda_{J}}\left(\tau_{n} B_{n J}\right) \mathrm{e}^{\tau_{n} B_{n, J-1}} \\
& \sum_{\lambda_{J}=0}^{\Lambda_{J}-1 \Lambda_{J-1}-1} \frac{1}{\lambda_{J-1}=0} \tau^{\lambda_{J-1} ! \lambda_{J} !} \tau_{n}^{\lambda_{J-1}+\lambda_{J}} B_{n J}^{\lambda_{J}} B_{n, J-1}^{\lambda_{J-1}} \\
& +\tau_{n}^{\Lambda_{J}}\left(\sum_{\lambda_{J}=0}^{\Lambda_{J}-1} \frac{1}{\lambda_{J} !} B_{n J}^{\lambda_{J}} B_{n, J-1}^{\Lambda_{J-1}} \varphi_{\Lambda_{J-1}}\left(\tau_{n} B_{n, J-1}\right)+B_{n J}^{\Lambda_{J}} \varphi_{\Lambda_{J}}\left(\tau_{n} B_{n J}\right) \mathrm{e}^{\tau_{n} B_{n, J-1}}\right)
\end{aligned}
$$


we point out that the range for the index $\lambda_{J-1}$ is chosen in dependence of the preceding index $\lambda_{J}$ such that the remainder involves the same power of the time increment, namely $\tau_{n}^{\Lambda_{J}}$. In order to indicate the dependence of the remainder on certain values of $A$ and products thereof, comprising a total of $\Lambda_{J}$ factors, we henceforth employ the short notation $\mathscr{O}\left(\tau_{n}^{\Lambda_{J}}, A^{\Lambda_{J}}\right)$; hence, the above relation rewrites as

$$
\mathrm{e}^{\tau_{n} B_{n J}} \mathrm{e}^{\tau_{n} B_{n, J-1}}=\sum_{\lambda_{J}=0}^{\Lambda_{J}-1} \sum_{\lambda_{J-1}=0}^{\Lambda_{J-1}-1} \frac{1}{\lambda_{J-1} ! \lambda_{J} !} \tau_{n}^{\lambda_{J-1}+\lambda_{J}} B_{n J}^{\lambda_{J}} B_{n, J-1}^{\lambda_{J-1}}+\mathscr{O}\left(\tau_{n}^{\Lambda_{J}}, A^{\Lambda_{J}}\right) .
$$

In an inductive manner, for any $j \in \mathscr{J}$ the expansion

$$
\begin{gathered}
\prod_{i=j+1}^{J} \mathrm{e}^{\tau_{n} B_{n i}}=\sum_{\lambda \in L_{j+1}} \frac{1}{\lambda !} \tau_{n}^{|\lambda|}\left(\prod_{i=j+1}^{J} B_{n i}^{\lambda_{i}}\right)+\mathscr{O}\left(\tau_{n}^{p-\ell-m-\kappa}, A^{p-\ell-m-\kappa}\right), \\
L_{j+1}=\left\{\lambda=\left(\lambda_{j+1}, \ldots, \lambda_{J}\right) \in \mathbb{N}^{J-j}: 0 \leqslant \lambda_{i} \leqslant \Lambda_{i}-1=p-\ell-m-\kappa-\sum_{\tilde{i}=i+1}^{J} \lambda_{i}-1 \text { for } j+1 \leqslant i \leqslant J\right\} ;
\end{gathered}
$$

follows; here, we set $|\lambda|=\lambda_{j+1}+\cdots+\lambda_{J}$ as well as $\lambda !=\lambda_{j+1} ! \cdots \lambda_{J} !$ for $\lambda=\left(\lambda_{j+1}, \ldots, \lambda_{J}\right) \in \mathbb{N}^{J-j}$. As a consequence, we obtain

$$
\begin{aligned}
& \delta_{n+1}=\delta_{n+1}^{(3)}+\mathscr{R}_{n+1}^{(1)}+\mathscr{R}_{n+1}^{(2)}+\mathscr{R}_{n+1}^{(3)}, \\
& \delta_{n+1}^{(3)}=\sum_{j=1}^{J} \sum_{k=1}^{K} \sum_{\substack{\ell=1 \\
\ell+m \leqslant p-1}}^{p-1} \sum_{m=0}^{p-2} \sum_{\kappa=0}^{p-\ell-m-1} \sum_{\lambda \in L_{j+1}} \frac{1}{\ell ! m ! \lambda !} a_{j k} \tau_{n}^{\ell+m+\kappa+|\lambda|+1} \\
& \times\left(\sum_{\mu=0}^{\ell+m} \frac{(\ell+m) !}{\mu !(\ell+m+\kappa+1-\mu) !} b_{j}^{\ell+m-\mu} d_{j-1}^{\mu}-\sum_{v=0}^{m} \frac{m !}{v !(m+\kappa+1-v) !} b_{j}^{m-v} c_{k}^{\ell} d_{j-1}^{v}\right) \\
& \times\left(\prod_{i=j+1}^{J} B_{n i}^{\lambda_{i}}\right) B_{n j}^{\kappa} A^{(\ell)}\left(t_{n}\right) u^{(m)}\left(t_{n}\right) ;
\end{aligned}
$$

the remainder $\mathscr{R}_{n+1}^{(3)}$ comprises certain compositions of the linear operators $B_{n j}$ for $j \in \mathscr{J}$ and can be cast into the form $\mathscr{O}\left(\tau_{n}^{p+1}, A^{(p)}, u^{(p-1)}\right)$. Employing the abbreviation

$$
\begin{aligned}
\mathscr{K}_{j \ell m}=\left\{\kappa=\left(\kappa_{j}, \ldots, \kappa_{J}\right) \in \mathbb{N}^{J+1-j}: 0 \leqslant \kappa_{j} \leqslant p-\ell-m-1,\right. \\
\left.0 \leqslant \kappa_{i} \leqslant p-\ell-m-\kappa_{j}-\sum_{\widetilde{i}=i+1}^{J} \kappa_{\tilde{i}}-1 \text { for } j+1 \leqslant i \leqslant J\right\},
\end{aligned}
$$


we may rewrite $\delta_{n+1}^{(3)}$ as follows

$$
\begin{aligned}
\delta_{n+1}^{(3)}=\sum_{j=1}^{J} & \sum_{k=1}^{K} \sum_{\substack{\ell=1 \\
\ell+m \leqslant p-1}}^{p-1} \sum_{m=0}^{p-2} \sum_{\kappa \in \mathscr{K}_{j \ell m}} \frac{\kappa_{j} !}{\ell ! m ! \kappa !} a_{j k} \tau_{n}^{\ell+m+|\kappa|+1} \\
& \times\left(\sum_{\mu=0}^{\ell+m} \frac{(\ell+m) !}{\mu !\left(\ell+m+\kappa_{j}+1-\mu\right) !} b_{j}^{\ell+m-\mu} d_{j-1}^{\mu}-\sum_{v=0}^{m} \frac{m !}{v !\left(m+\kappa_{j}+1-v\right) !} b_{j}^{m-v} c_{k}^{\ell} d_{j-1}^{v}\right) \\
& \times\left(\prod_{i=j}^{J} B_{n i}^{\kappa_{i}}\right) A^{(\ell)}\left(t_{n}\right) u^{(m)}\left(t_{n}\right) .
\end{aligned}
$$

FINAL EXPANSION In a final expansion step, we employ the Taylor series expansion

$$
B_{n i}=\sum_{k=1}^{K} a_{i k} A\left(t_{n}+c_{k} \tau_{n}\right)=\sum_{k=1}^{K} \sum_{\lambda=0}^{\Lambda-1} \frac{1}{\lambda !} a_{i k}\left(c_{k} \tau_{n}\right)^{\lambda} A^{(\lambda)}\left(t_{n}\right)+\mathscr{O}\left(\tau_{n}^{\Lambda}\right)=\sum_{\lambda=0}^{\Lambda-1} \frac{1}{\lambda !} \gamma_{i \lambda} \tau_{n}^{\lambda} A^{(\lambda)}\left(t_{n}\right)+\mathscr{O}\left(\tau_{n}^{\Lambda}\right)
$$

see also (2.3) for the definition of $\gamma_{i \lambda}$. For notational simplicity, we do not specify the remainder terms arising in this expansion step; again, they can be cast into the form $\mathscr{O}\left(\tau_{n}^{p+1}, A^{(p)}, u^{(p-1)}\right)$. Analogously to before, we choose the upper indices such that the same power $\Lambda_{\kappa_{J}}^{(J)}=p-\ell-m-|\kappa|$ occurs; proceeding by induction, setting $\Lambda_{\kappa_{J}-1}^{(J)}=\Lambda_{\kappa_{J}}^{(J)}-\lambda_{\kappa_{J}}^{(J)}$ etc., this yields the expansion

$$
\begin{aligned}
& \prod_{i=j}^{J} B_{n i}^{\kappa_{i}}=B_{n J} B_{n J}^{\kappa_{J}-1}\left(\prod_{i=j}^{J-1} B_{n i}^{\kappa_{i}}\right) \\
& =\sum_{\lambda_{\kappa_{J}}^{(J)}=0}^{\Lambda_{\kappa_{J}}^{(J)}-1} \frac{1}{\lambda_{\kappa_{J}}^{(J)} !} \gamma_{J \lambda_{\kappa_{J}}^{(J)}} \tau_{n}^{\lambda_{\kappa_{J}}^{(J)}} A^{\left(\lambda_{\kappa_{J}}^{(J)}\right)}\left(t_{n}\right) B_{n J}^{\kappa_{J}-1}\left(\prod_{i=j}^{J-1} B_{n i}^{\kappa_{i}}\right)+\mathscr{O}\left(\tau_{n}^{\Lambda_{\kappa_{J}}^{(J)}}\right) \\
& =\sum_{\lambda_{\kappa_{J}}^{(J)}=0}^{\Lambda_{\kappa_{J}}^{(J)}-1} \sum_{\lambda_{\kappa_{J}-1}^{(J)}=0}^{\Lambda_{\kappa_{J}-1}^{(J)}-1} \frac{1}{\lambda_{\kappa_{J}-1}^{(J)} ! \lambda_{\kappa_{J}}^{(J)} !} \gamma_{J \lambda_{\kappa_{J}-1}^{(J)}} \gamma_{J \lambda_{\kappa_{J}}^{(J)}} \tau_{n}^{\lambda_{\kappa_{J}-1}^{(J)}+\lambda_{\kappa_{J}}^{(J)}} A^{\left(\lambda_{\kappa_{J}}^{(J)}\right)}\left(t_{n}\right) A^{\left(\lambda_{\kappa_{J}-1}^{(J)}\right)}\left(t_{n}\right) B_{n J}^{\kappa_{J}-2}\left(\prod_{i=j}^{J-1} B_{n i}^{\kappa_{i}}\right) \\
& +\mathscr{O}\left(\tau_{n}^{\Lambda_{\kappa_{J}}^{(J)}}\right) \\
& =\sum_{\lambda \in \mathscr{L}_{j \ell m \kappa}} \frac{1}{\lambda !} \gamma_{\lambda} \tau_{n}^{|\lambda|} A^{(\lambda)}\left(t_{n}\right)+\mathscr{O}\left(\tau_{n}^{\Lambda_{\kappa_{J}}^{(J)}}\right) ;
\end{aligned}
$$

here, for $j \in \mathscr{J}$, we employ the abbreviations

$$
\begin{gathered}
\lambda^{(i)}=\left(\lambda_{1}^{(i)}, \ldots, \lambda_{\kappa_{i}}^{(i)}\right) \in \mathbb{N}^{\kappa_{i}}, \quad j \leqslant i \leqslant J, \quad \lambda=\left(\lambda^{(j)}, \ldots, \lambda^{(J)}\right) \in \mathbb{N}^{|\kappa|} \\
|\lambda|=\sum_{i=j}^{J}\left|\lambda^{(i)}\right|=\sum_{i=j}^{J} \sum_{\eta_{i}=1}^{\kappa_{i}} \lambda_{\eta_{i}}^{(i)}, \quad \gamma_{\lambda}=\prod_{i=j}^{J} \prod_{\eta_{i}=1}^{\kappa_{i}} \gamma_{i \lambda_{\eta_{i}}^{(i)}}, \\
A^{(\lambda)}=A^{\left(\lambda_{\kappa_{J}}^{(J)}\right)}\left(t_{n}\right) \cdots A^{\left(\lambda_{1}^{(J)}\right)}\left(t_{n}\right) \cdots A^{\left(\lambda_{\kappa_{j}}^{(j)}\right)}\left(t_{n}\right) \cdots A^{\left(\lambda_{1}^{(j)}\right)}\left(t_{n}\right),
\end{gathered}
$$


30 of 34

S. BLANES, F. CASAS, M. THALHAMMER

$$
\begin{aligned}
\mathscr{L}_{j \ell m \kappa}=\{ & \lambda=\in \mathbb{N}^{|\kappa|}: 0 \leqslant \lambda_{\eta_{i}}^{(i)} \leqslant p-\ell-m-|\kappa|-\sum_{\widetilde{i}=i+1}^{J}\left|\lambda^{(\widetilde{i})}\right|-\sum_{\widetilde{\eta}=\eta_{i}+1}^{\kappa_{i}} \lambda_{\widetilde{\eta}}^{(i)}-1 \\
& \text { for } \left.1 \leqslant \eta_{i} \leqslant \kappa_{i} \text { and } j \leqslant i \leqslant J\right\} .
\end{aligned}
$$

Clearly, the summation over the subindex $\lambda^{(i)}$ does not arise whenever $\kappa_{i}=0$ and, in particular, the summation over the index $\lambda$ does not arise whenever $|\kappa|=0$. Altogether, we obtain the local error expansion (3.10), where the remainder is of the form

$$
\mathscr{R}_{n+1}=\mathscr{R}_{n+1}^{(1)}+\mathscr{R}_{n+1}^{(2)}+\mathscr{R}_{n+1}^{(3)}+\mathscr{R}_{n+1}^{(4)}=\mathscr{O}\left(\tau_{n}^{p+1}, A^{(p)}, u^{(p-1)}\right) .
$$

\section{B. Illustration}

In this section, we illustrate our approach for the derivation of (3.10) on the basis of a fourth-order CFQM exponential integrator involving two nodes and two exponentials per time step. That is, setting $p=4$ as well as $J=K=2$, relation (3.9) involves the index sets

$$
\begin{gathered}
\ell+m=1: \quad(\ell, m)=(1,0), \\
\mathscr{K}_{110}=\{(0,0),(0,1),(0,2),(1,0),(1,1),(2,0)\}, \quad \mathscr{K}_{210}=\{0,1,2\}, \\
\ell+m=2: \quad(\ell, m) \in\{(1,1),(2,0)\}, \\
\mathscr{K}_{111}=\mathscr{K}_{120}=\{(0,0),(0,1),(1,0)\}, \quad \mathscr{K}_{211}=\mathscr{K}_{220}=\{0,1\}, \\
\ell+m=3: \quad(\ell, m) \in\{(1,2),(2,1),(3,0)\}, \\
\mathscr{K}_{112}=\mathscr{K}_{121}=\mathscr{K}_{130}=\{(0,0)\}, \quad \mathscr{K}_{212}=\mathscr{K}_{221}=\mathscr{K}_{230}=\{0\},
\end{gathered}
$$


and thus simplifies to

$$
\begin{aligned}
& \delta_{n+1}=\sum_{j=1}^{2} \sum_{k=1}^{2} \sum_{\substack{\ell=1 \\
\ell+m \leqslant 3}}^{2} \sum_{m=0} \sum_{\kappa \in \mathscr{K}_{j \ell m}} c_{j k \ell m \kappa} \tau_{n}^{\ell+m+|\kappa|+1}\left(\prod_{i=j}^{2} B_{n i}^{\kappa_{i}}\right) A^{(\ell)}\left(t_{n}\right) u^{(m)}\left(t_{n}\right)+\mathscr{O}\left(\tau_{n}^{5}, A^{(4)}, u^{(3)}\right) \\
& =\left(c_{11100}+c_{12100}+c_{21100}+c_{22100}\right) \tau_{n}^{2} A^{\prime}\left(t_{n}\right) u\left(t_{n}\right) \\
& +\left(c_{1110(1,0)}+c_{1210(1,0)}\right) \tau_{n}^{3} B_{n 1} A^{\prime}\left(t_{n}\right) u\left(t_{n}\right) \\
& +\left(c_{1110(0,1)}+c_{1210(0,1)}+c_{21101}+c_{22101}\right) \tau_{n}^{3} B_{n 2} A^{\prime}\left(t_{n}\right) u\left(t_{n}\right) \\
& +\left(c_{11200}+c_{12200}+c_{21200}+c_{22200}\right) \tau_{n}^{3} A^{\prime \prime}\left(t_{n}\right) u\left(t_{n}\right) \\
& +\left(c_{11110}+c_{12110}+c_{21110}+c_{22110}\right) \tau_{n}^{3} A^{\prime}\left(t_{n}\right) u^{\prime}\left(t_{n}\right) \\
& +\left(c_{1110(2,0)}+c_{1210(2,0)}\right) \tau_{n}^{4} B_{n 1}^{2} A^{\prime}\left(t_{n}\right) u\left(t_{n}\right) \\
& +\left(c_{1110(0,2)}+c_{1210(0,2)}\right) \tau_{n}^{4} B_{n 2}^{2} A^{\prime}\left(t_{n}\right) u\left(t_{n}\right) \\
& +\left(c_{1110(1,1)}+c_{1210(1,1)}\right) \tau_{n}^{4} B_{n 2} B_{n 1} A^{\prime}\left(t_{n}\right) u\left(t_{n}\right) \\
& +\left(c_{21102}+c_{22102}\right) \tau_{n}^{4} B_{n 2}^{2} A^{\prime}\left(t_{n}\right) u\left(t_{n}\right) \\
& +\left(c_{1120(1,0)}+c_{1220(1,0)}\right) \tau_{n}^{4} B_{n 1} A^{\prime \prime}\left(t_{n}\right) u\left(t_{n}\right) \\
& +\left(c_{1120(0,1)}+c_{1220(0,1)}+c_{21201}+c_{22201}\right) \tau_{n}^{4} B_{n 2} A^{\prime \prime}\left(t_{n}\right) u\left(t_{n}\right) \\
& +\left(c_{11300}+c_{12300}+c_{21300}+c_{22300}\right) \tau_{n}^{4} A^{\prime \prime \prime}\left(t_{n}\right) u\left(t_{n}\right) \\
& +\left(c_{1111(1,0)}+c_{1211(1,0)}\right) \tau_{n}^{4} B_{n 1} A^{\prime}\left(t_{n}\right) u^{\prime}\left(t_{n}\right) \\
& +\left(c_{1111(0,1)}+c_{1211(0,1)}+c_{21111}+c_{22111}\right) \tau_{n}^{4} B_{n 2} A^{\prime}\left(t_{n}\right) u^{\prime}\left(t_{n}\right) \\
& +\left(c_{11210}+c_{12210}+c_{21210}+c_{22210}\right) \tau_{n}^{4} A^{\prime \prime}\left(t_{n}\right) u^{\prime}\left(t_{n}\right) \\
& +\left(c_{11120}+c_{12120}+c_{21120}+c_{22120}\right) \tau_{n}^{4} A^{\prime}\left(t_{n}\right) u^{\prime \prime}\left(t_{n}\right) \\
& +\mathscr{O}\left(\tau_{n}^{5}, A^{(4)}, u^{(3)}\right) \text {. }
\end{aligned}
$$

In the present situation, we employ the stepwise expansion

$$
\begin{aligned}
B_{n i} & =b_{i} A\left(t_{n}\right)+\mathscr{O}\left(\tau_{n}\right) \\
& =b_{i} A\left(t_{n}\right)+\gamma_{i 1} \tau_{n} A^{\prime}\left(t_{n}\right)+\mathscr{O}\left(\tau_{n}^{2}\right) \\
& =b_{i} A\left(t_{n}\right)+\gamma_{i 1} \tau_{n} A^{\prime}\left(t_{n}\right)+\frac{1}{2} \gamma_{i 2} \tau_{n}^{2} A^{\prime \prime}\left(t_{n}\right)+\mathscr{O}\left(\tau_{n}^{3}\right),
\end{aligned}
$$

and, as a consequence, we obtain the following local error expansion

$$
\delta_{n+1}=C^{(2)} \tau_{n}^{2}+C^{(3)} \tau_{n}^{3}+C^{(4)} \tau_{n}^{4}+\mathscr{O}\left(\tau_{n}^{5}, A^{(4)}, u^{(3)}\right)
$$

comprising the compositions

$$
\begin{aligned}
C^{(2)}= & C_{10} A^{\prime}\left(t_{n}\right) u\left(t_{n}\right), \\
C^{(3)}= & C_{010} A\left(t_{n}\right) A^{\prime}\left(t_{n}\right) u\left(t_{n}\right)+C_{20} A^{\prime \prime}\left(t_{n}\right) u\left(t_{n}\right)+C_{11} A^{\prime}\left(t_{n}\right) u^{\prime}\left(t_{n}\right), \\
C^{(4)}= & C_{110} A^{\prime}\left(t_{n}\right) A^{\prime}\left(t_{n}\right) u\left(t_{n}\right)+C_{0010} A\left(t_{n}\right) A\left(t_{n}\right) A^{\prime}\left(t_{n}\right) u\left(t_{n}\right)+C_{020} A\left(t_{n}\right) A^{\prime \prime}\left(t_{n}\right) u\left(t_{n}\right) \\
& \quad+C_{30} A^{\prime \prime \prime}\left(t_{n}\right) u\left(t_{n}\right)+C_{011} A\left(t_{n}\right) A^{\prime}\left(t_{n}\right) u^{\prime}\left(t_{n}\right)+C_{21} A^{\prime \prime}\left(t_{n}\right) u^{\prime}\left(t_{n}\right)+C_{12} A^{\prime}\left(t_{n}\right) u^{\prime \prime}\left(t_{n}\right),
\end{aligned}
$$


where

$$
\begin{aligned}
C_{10}= & c_{11100}+c_{12100}+c_{21100}+c_{22100} \\
C_{010}= & b_{1}\left(c_{1110(1,0)}+c_{1210(1,0)}\right)+b_{2}\left(c_{1110(0,1)}+c_{1210(0,1)}+c_{21101}+c_{22101}\right) \\
C_{20}= & c_{11200}+c_{12200}+c_{21200}+c_{22200} \\
C_{11}= & c_{11110}+c_{12110}+c_{21110}+c_{22110} \\
C_{110}= & \gamma_{11}\left(c_{1110(1,0)}+c_{1210(1,0)}\right)+\gamma_{21}\left(c_{1110(0,1)}+c_{1210(0,1)}+c_{21101}+c_{22101}\right) \\
C_{0010}= & b_{1}^{2}\left(c_{1110(2,0)}+c_{1210(2,0)}\right)+b_{1} b_{2}\left(c_{1110(1,1)}+c_{1210(1,1)}\right) \\
& \quad+b_{2}^{2}\left(c_{1110(0,2)}+c_{1210(0,2)}+c_{21102}+c_{22102}\right) \\
C_{020}= & b_{1}\left(c_{1120(1,0)}+c_{1220(1,0)}\right)+b_{2}\left(c_{1120(0,1)}+c_{1220(0,1)}+c_{21201}+c_{22201}\right) \\
C_{30}= & c_{11300}+c_{12300}+c_{21300}+c_{22300} \\
C_{011}= & b_{1}\left(c_{1111(1,0)}+c_{1211(1,0)}\right)+b_{2}\left(c_{1111(0,1)}+c_{1211(0,1)}+c_{21111}+c_{22111}\right) \\
C_{21}= & c_{11210}+c_{12210}+c_{21210}+c_{22210} \\
C_{12}= & c_{11120}+c_{12120}+c_{21120}+c_{22120} .
\end{aligned}
$$

We note that the operators $A(t)$ and $A^{\prime}(t)$ do not commute in general; thus, using the differential equation and inserting the relations $u^{\prime}(t)=A(t) u(t)$ as well as $u^{\prime \prime}(t)=A^{\prime}(t) u(t)+A(t) A(t) u(t)$ does not lead to a further simplification. The requirement

$$
\delta_{n+1}=\mathscr{O}\left(\tau_{n}^{5}\right)
$$

implies that the quantities

$$
C_{10}, C_{010}, C_{20}, C_{11}, C_{110}, C_{0010}, C_{020}, C_{30}, C_{011}, C_{21}, C_{12},
$$

involving the method coefficients $a_{j k}$ and $c_{k}$ for $j=1,2$ and $k=1,2$ should vanish; this set of (redundant) order conditions possesses a uniquely determined solution, the CFQM exponential integrator (2.4) based on two Gaussian nodes. For completeness, the order conditions are included in a simple MAPLEimplementation.

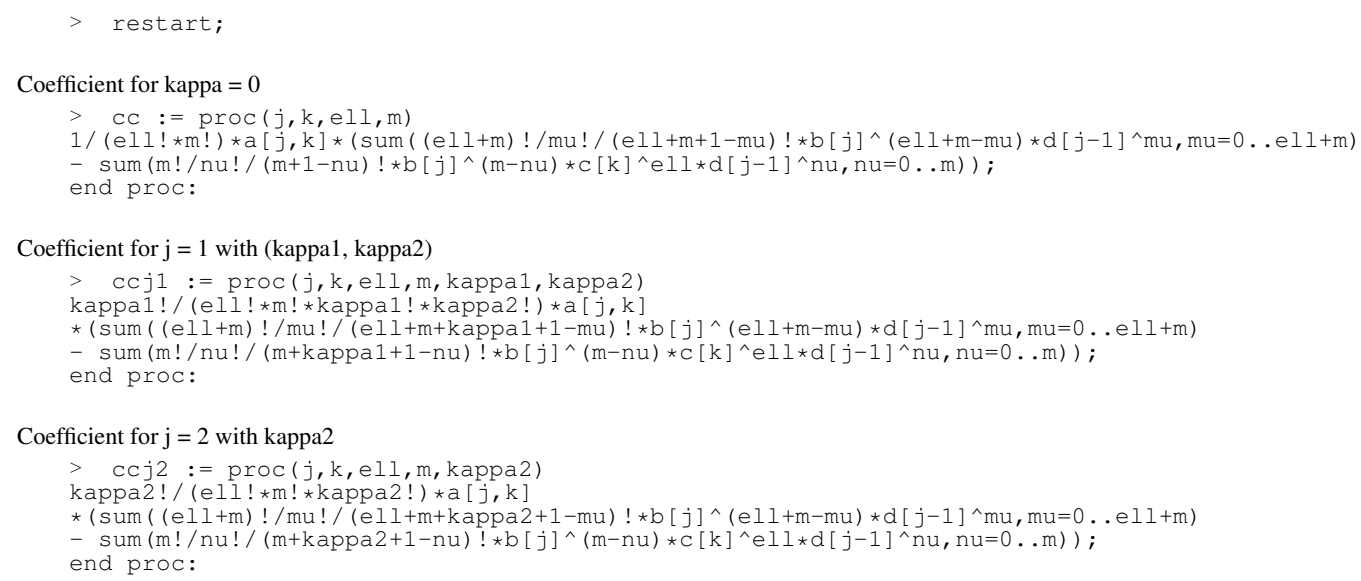




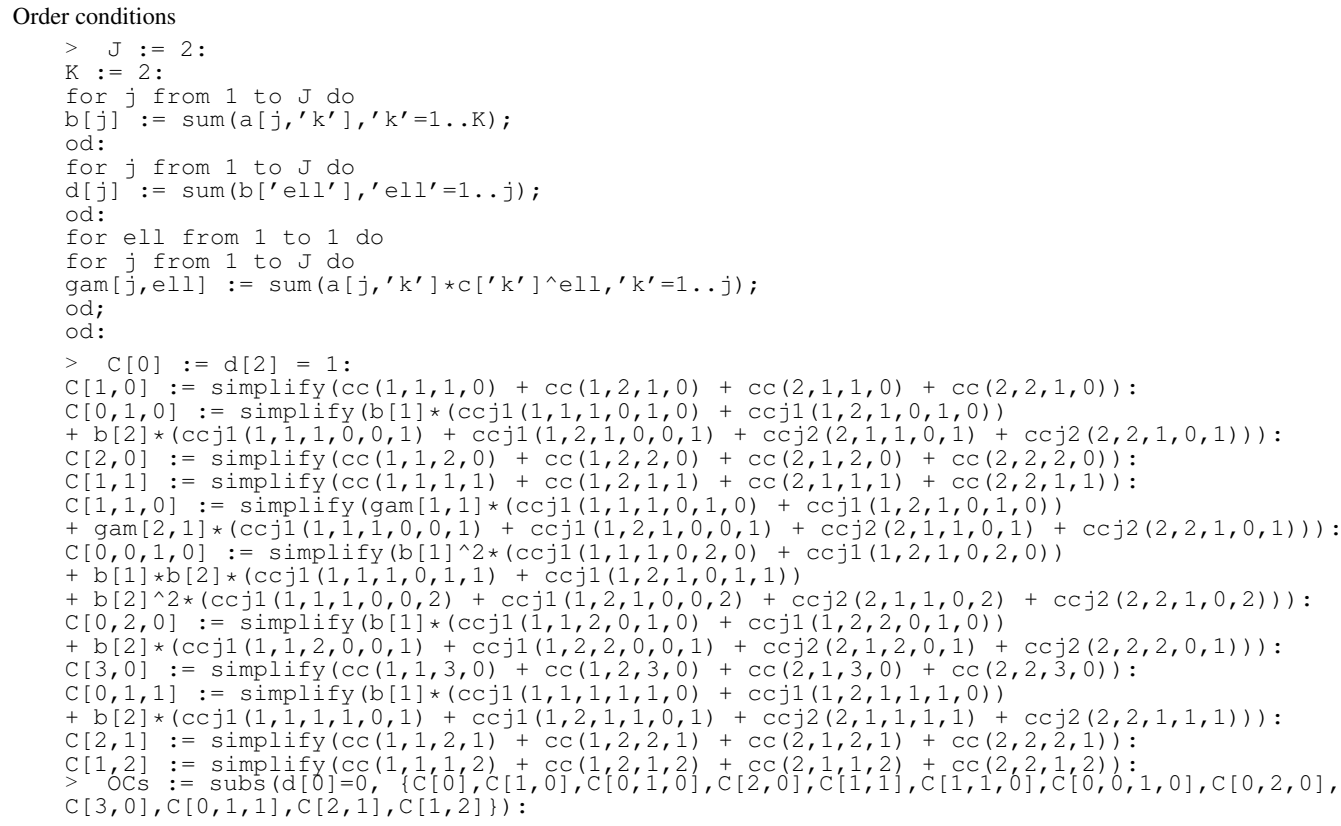

Solution of order conditions

$>$ allvalues(solve(OCs))[2];

$$
\left\{a_{1,1}=\frac{1}{4}+\frac{\sqrt{3}}{6}, a_{1,2}=\frac{1}{4}-\frac{\sqrt{3}}{6}, a_{2,1}=\frac{1}{4}-\frac{\sqrt{3}}{6}, a_{2,2}=\frac{1}{4}+\frac{\sqrt{3}}{6}, c_{1}=\frac{1}{2}-\frac{\sqrt{3}}{6}, c_{2}=\frac{1}{2}+\frac{\sqrt{3}}{6}\right\}
$$

\section{References}

A. Alvermann, H. Fehske, High-order commutator-free exponential time-propagation of driven quantum systems. J. Comp. Phys. 230/15 (2011) 5930-5956.

A. Alvermann, H. Fehske, P. B. Littlewood, Numerical time propagation of quantum systems in radiation fields. New. J. Phys. 14 (2012) 105008 (22pp).

PH. BADER, A. ISERLES, K. KROPIELNICKA, P. SINGH, Efficient methods for linear Schrödinger equation in the semiclassical regime with time-dependent potential. Proc. R. Soc. A 472 (2016) 20150733.

S. Blanes, F. CASAS, M. Thalhammer, High-order commutator-free quasi-Magnus exponential integrators for non-autonomous linear evolution equations. In preparation.

S. Blanes, F. CASAs, J. A. OteO, J. Ros, The Magnus expansion and some of its applications. Physics Reports 470/5-6 (2009) 151-238.

S. BLANES, P. C. MOAN, Fourth-and sixth-order commutator-free Magnus integrators for linear and non-linear dynamical systems. App. Num. Math. 56/12 (2006) 1519-1537.

H. BRUnNner, P.J. VAN DER HOUWEn, The numerical solution of Volterra equations. CWI Monographs 3. North-Holland, Amsterdam, 1986.

E. Celledoni, A. Marthinsen, B. Owren, Commutator-free Lie group methods. Future Generation Computer Systems 19 (2003) 341-352.

P. E. CRouch AND R. Grossman, Numerical integration of ordinary differential equations on manifolds. J. Nonlinear Sci. 3 (1993) 1-33.

K. J. Engel, R. Nagel, One-Parameter Semigroups for Linear Evolution Equations. Springer, New York, 2000.

D. Henry, Geometric Theory of Semilinear Parabolic Equations. Lecture Notes in Mathematics 840, Springer, Berlin, 1981. 
M. Hochbruck, CH. Lubich, On Krylov subspace approximations to the matrix exponential operator. SIAM J. Numer. Anal. 34 (1997) 1911-1925.

M. Hochbruck, CH. LubiCH, On Magnus integrators for time-dependent Schrödinger equations. SIAM J. Numer. Anal. 41/3 (2003) 945-963.

A. LunARd, Analytic Semigroups and Optimal Regularity in Parabolic Problems. Birkhäuser, Basel, 1995.

W. Magnus, On the exponential solution of a differential equation for a linear operator. Comm. Pure Appl. Math. 7 (1954) 649-673.

C. Moler, Ch. VAn LoAn, Nineteen dubious ways to compute the exponential of a matrix, twenty-five years later. SIAM Rev. 45/1 (2003) 3-49.

B. OWREN, Order conditions for commutator-free Lie group methods. J. Phys. A 39/19 (2006) 5585-5599.

C. V. PAO, Numerical methods for time-periodic solutions of nonlinear parabolic boundary value problems. SIAM J. Numer. Anal. 39/2 (2001) 647-667.

A. PAZY, Semigroups of Linear Operators and Applications to Partial Differential Equations. Springer, New York, 1983.

R. B. SIDJE, Expokit: a software package for computing matrix exponentials. ACM Trans. Math. Software 24 (1998) 130-156.

M. THALHAMMER, A fourth-order commutator-free exponential integrator for nonautonomous differential equations. SIAM J. Numer. Anal. 44/2 (2006) 851-864.

M. THALHAMMER, Convergence analysis of high-order time-splitting pseudo-spectral methods for nonlinear Schrödinger equations. SIAM J. Numer. Anal. 50/6 (2012) 3231-3258.

H. TANABE, Equations of Evolution. Pitman, London, 1979. 